Review Article

\title{
Diversity of Ecosystem Types in India: A Review
}

\author{
J S SINGH ${ }^{1}$ and R K CHATURVEDI*,2 \\ ${ }^{1}$ Ecosystems Analysis laboratory, Department of Botany, Banaras Hindu University, Varanasi 221005, \\ India \\ ${ }^{2}$ Centre for Integrative Conservation, Xishuangbanna Tropical Botanical Garden, Chinese Academy of \\ Sciences, Menglun, Mengla, Yunnan 666303, China
}

(Received on 15 May 2016; Revised on 20 October 2016; Accepted on 21 October 2016)

\begin{abstract}
India is home to an unusually large number of endemic species and ranks sixth among the 12 megabiodiversity centers of the world. Four terrestrial biodiversity hot spots (Himalaya, Indo-Burma, Western Ghats and Sri Lanka, and Sundaland) partly lie in India. The 27 distinct biogeographic provinces, and the variety of life zones and floral groups result in equally diverse vegetation and ecosystem types. The forest ecosystems are markedly diverse and have been classified into six 'major groups' ranging from tropical to alpine, 16 'groups' and more than 200 'group categories'. The grassland ecosystems are categorized into five major grass cover types. There are 1193 wetlands covering a total area of 3.9 million hectares in 274 districts. Total area of coral reefs comprises 2,375 sq km. India is one of the eight Vavilov's centers of origin of cultivated plants in the world and has twenty distinct agro-ecosystems, characterized by variations in edaphic, climatic and geographic features, and consequently a diverse cropping pattern. In this article we give an overview of the vast array of broad ecosystem types that occur in India. We also briefly discuss the human impacts on the health of country's ecosystems and the ecosystem conservation scenario.
\end{abstract}

Keywords: India; Ecosystem Diversity; Forests; Grasslands; Wetlands; Agricultural Zones

\section{Introduction}

India is situated north of the equator between $66^{\circ} \mathrm{E}$ to $98^{\circ} \mathrm{E}$ long. and $8^{\circ} \mathrm{N}$ to $36^{\circ} \mathrm{N}$ lat. It is bordered by Nepal, China and Bhutan in the north; Bangladesh and Myanmar in the east; the Bay of Bengal in the south east; the Indian Ocean in the south; the Arabian Sea in the west; and Pakistan in the north-west. Total land area of India is 3.3 million square kilometers and it is the seventh largest country in the world. It is 2,933 kms wide and 3,214 kms long. In the north, Himalayan ranges separate the Indian sub-continent from the rest of Asia. Down towards south lies the Indo-Gangetic plains which is further crossed over by the Vindhyachal mountains. Next lies the Deccan Peninsula which is bounded by Arabian sea to the south-west and the Bay of Bengal to the south-east. The southern-most tip of the country projects into the Indian Ocean. Mountains cover an area of around
$100 \mathrm{mha}$, arid and semi-arid zones are spread over 30 mha and the coastline is about $8000 \mathrm{~km}$ long (MoEF, 2009). The three great rivers of Northern India - the Indus, the Ganges and the Brahmaputra, have originated from the Himalaya. The vast area of the Indo-Gangetic plains between the Himalaya and the Deccan plateau has been filled up by the alluvium transported from the Himalaya by the great river system. The Mahanadi, Godavari, Krishna, Kaveri and the Pennar in the eastern coast and the Narmada, Tapti, Sharavati, Netravati, Bharatapuzha, Periyar and the Pamba in the west coast have created a variety of deltas and flood-plains. India represents great geological, geomorphological, climatic, biotic and cultural diversity. The vast diversity of climatic features (tropical to arctic) and habitats (plains, wetlands and mountains) has led to a wide variety of flora and fauna, which form diverse ecosystems.

*Author for Correspondence: E-mail: ravikantchaturvedi10@gmail.com; ravi@xtbg.ac.cn 
In this article we give an overview of the vast array of broad ecosystem types that occur in India.

\section{Biogeographical Regions and Holdridge Life- zones}

India is represented by two 'Realms'- Palearctic Realm (Himalayan region) and Malayan Realm (rest of the sub-continent), and ten biogeographic regions and 27 biogeographic provinces (Fig. 1, Table 1). The geographical analysis (Roy et al., 2006) reveals 19 Holdridge life-zones, seven biomes and 19 sub-biomes (Fig. 2). The biomes, as per Roy et al. (2006) are (i) Tropical Rain Forest, (ii) Tropical Wet Forest, (iii) Tropical Moist Forest, (iv) Tropical Dry Forest, (v) Tropical Thorn Woodland, (vi) Tropical Desert and (vii) Himalayan Temperate Tundra.

India is home to an unusually large number of endemic species and ranks sixth among the 12 mega biodiversity centers of the world. Four terrestrial biodiversity hot spots (Himalaya, Indo-Burma, Western Ghats and Sri Lanka, and Sundaland) partly lie in India. In terms of plant diversity, India ranks tenth in the world and fourth in Asia. India has over 45,500 plant species, which is nearly $11 \%$ of the world's known floral diversity. There are remote geographical areas which are yet to be comprehensively explored; and many organisms especially in lower groups such as bacteria, fungi, algae, lichens and bryophytes are yet to be described. New plant species, however, are continually being discovered in the country. For example, 41 plant taxa were discovered from diverse bio-geographic zones of India during 2007 by Botanical Survey of India (BSI) and other researchers. Similarly in cryptogams, the National Botanical Research Institute (NBRI), Lucknow described 11 new species during 2007-08. Under the All India Coordinated Project for capacity building in Taxonomy (AICOPTAX), funded by the Ministry of Environment and Forests (MoEF), 493

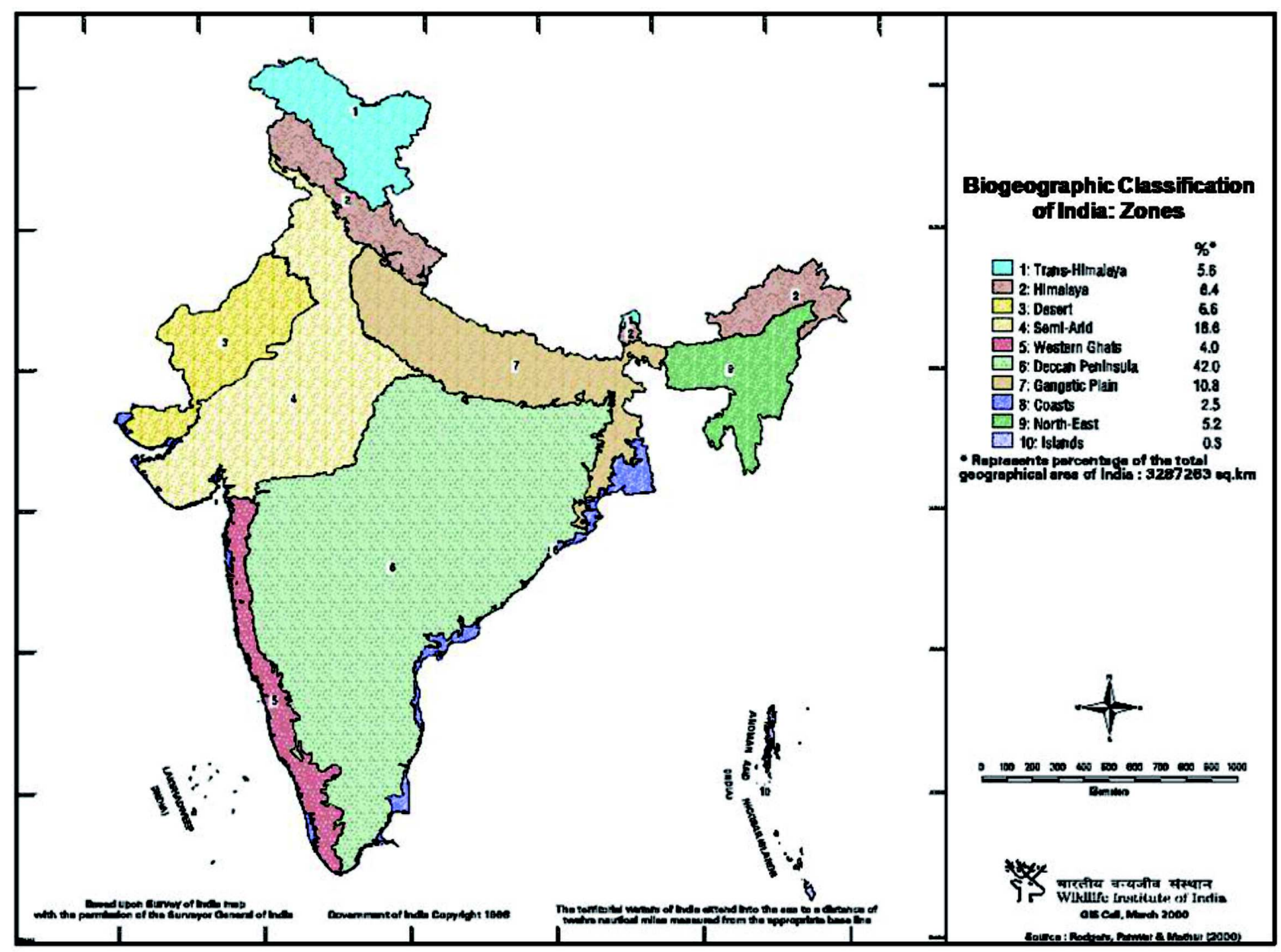

Fig. 1: Biogeographic zones of India (Source: Rodgers et al., 2000) 
Table 1: Biogeographic zones of India (Source: MoEF, 2009)

\begin{tabular}{|c|c|c|c|}
\hline S.No. & Biogeographic Zones & Biogeographic Provinces & $\%$ of geographical area \\
\hline \multirow[t]{3}{*}{1.} & \multirow[t]{3}{*}{ Trans Himalaya } & 1A: Himalaya - Ladakh Mountains & 3.3 \\
\hline & & 1B: Himalaya -Tibetan Plateau & 2.2 \\
\hline & & 1C: Trans - Himalaya Sikkim & $<0.1$ \\
\hline \multirow[t]{4}{*}{2.} & \multirow[t]{4}{*}{ The Himalaya } & 2A: Himalaya - North West Himalaya & 2.1 \\
\hline & & 2B: Himalaya - West Himalaya & 1.6 \\
\hline & & 2C: Himalaya - Central Himalaya & 0.2 \\
\hline & & 2D: Himalaya - East Himalaya & 2.5 \\
\hline \multirow[t]{2}{*}{3.} & \multirow[t]{2}{*}{ The Indian Desert } & 3A: Desert - Thar & 5.4 \\
\hline & & 3B: Desert - Katchchh & 1.1 \\
\hline \multirow[t]{2}{*}{4.} & \multirow[t]{2}{*}{ The Semi Arid } & 4A: Semi - Arid - Punjab Plains & 3.7 \\
\hline & & 4B: Semi - Arid - Gujarat, Rajputana & 12.9 \\
\hline \multirow[t]{2}{*}{5.} & \multirow[t]{2}{*}{ The Western Ghats } & 5A: Western Ghats - Malabar Plains & 2.0 \\
\hline & & 5B: Western Ghats -Western Ghats Mountains & 2.0 \\
\hline \multirow[t]{5}{*}{6.} & \multirow[t]{5}{*}{ The Deccan Peninsula } & 6A: Deccan Peninsular - Central Highlands & 7.3 \\
\hline & & 6B: Deccan Peninsular - Chotta Nagpur & 5.4 \\
\hline & & 6C: Deccan Peninsular - Eastern Highlands & 6.3 \\
\hline & & 6D: Deccan Peninsular - Central Plateau & 12.5 \\
\hline & & 6E: Deccan Peninsular - Deccan South & 10.4 \\
\hline \multirow[t]{2}{*}{7.} & \multirow[t]{2}{*}{ The Gangetic Plains } & 7A: Gangetic Plain - Upper Gangetic Plains & 6.3 \\
\hline & & 7B: Gangetic Plain - Lower Gangetic Plains & 4.5 \\
\hline \multirow[t]{3}{*}{8.} & \multirow[t]{3}{*}{ The Coasts } & 8A: Coasts - West Coast & 0.6 \\
\hline & & 8B: Coasts - East Coast & 1.9 \\
\hline & & 8C: Coasts - Lakshdweep & $<0.1$ \\
\hline \multirow[t]{2}{*}{9.} & \multirow[t]{2}{*}{ Northeast India } & 9A: North - East - Brahamputra Valley & 2.0 \\
\hline & & 9B: North - East - North East Hills & 3.2 \\
\hline \multirow[t]{2}{*}{10.} & \multirow[t]{2}{*}{ Islands } & 10A: Islands - Andamans & 0.2 \\
\hline & & 10B: Islands - Nicobars & 0.1 \\
\hline
\end{tabular}

taxa new to science have been discovered (MoEF, 2009). Important floral groups found in India are summarized in Table 2, and important wild fauna in the 10 biogeographic regions are listed in Table 3.

The 27 distinct biogeographic provinces, the variety of life-zones and floral groups result in equally diverse vegetation and ecosystem types. The broad ecosystem types are briefly described below.

\section{Forest Ecosystems}

According to the latest report of the forest survey of India (FSI, 2016), forest and tree cover of the country in 2015 was $702979 \mathrm{~km}^{2}$ or $24.16 \%$ of the geographical area $(21.34 \%$ forest cover $+2.82 \%$ tree cover). Of the total forest cover, very dense forest (canopy cover $>70 \%$ ) was only $2.5 \%$ of the geographical area, moderately dense forest (canopy cover 40-70\%) occupied the maximum area followed by open forest (canopy cover 10-40\%) There is an additional $1.28 \%$ of scrub vegetation. The forest ecosystems are markedly diverse. Champion and Seth (1968) classified India's forests into six 'major groups' ranging from tropical to alpine, 16 'groups' and more than 200 'group categories' (Table 4). According to Champion and Seth's classification, forest type is a unit of vegetation having physiognomic and structural features so pronounced that it appears distinct from 

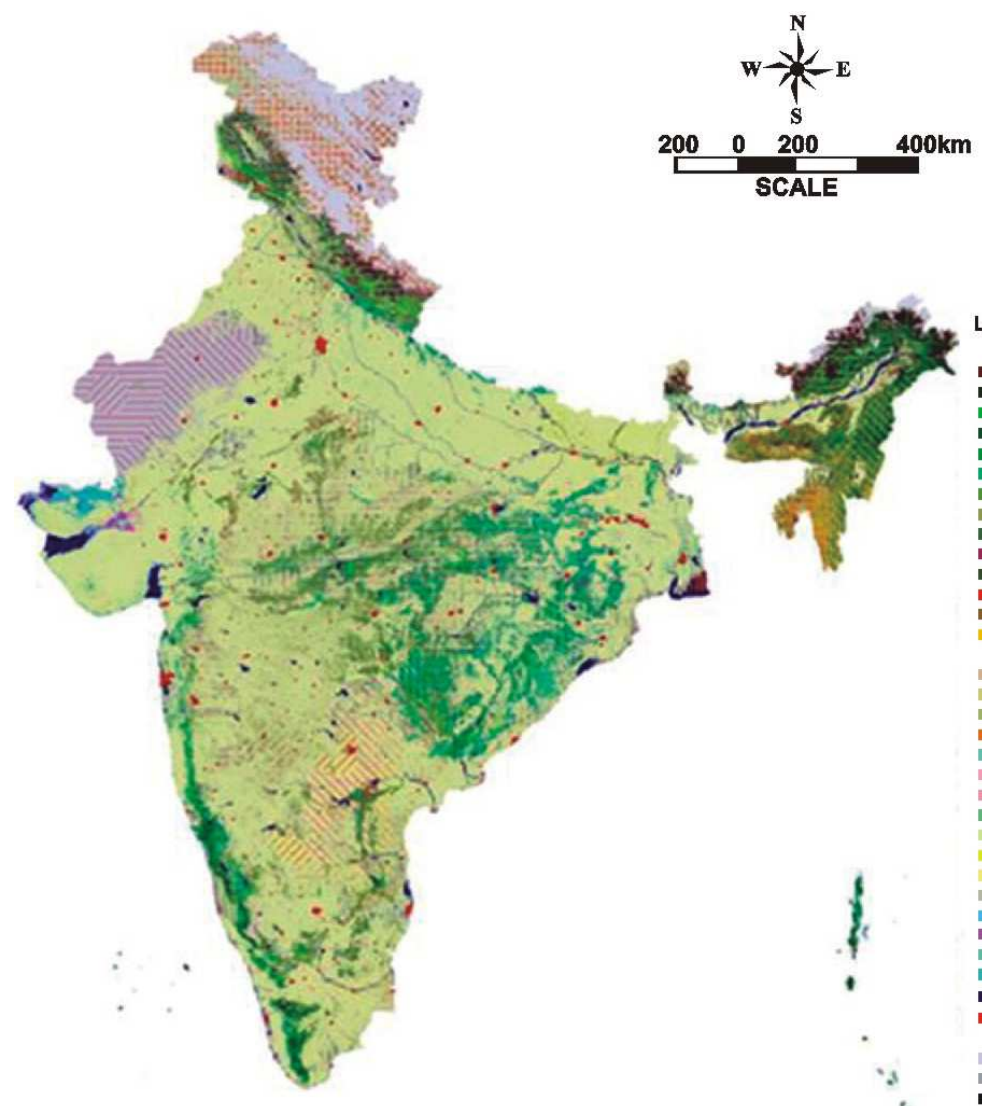

Biome Map

(Llfe zones Intersect with Vegetatlon cover type dellneate the Blome of Indla)

Fig. 2: Biome map of India (Source: Roy et al., 2006)

other units. Each forest group usually has a southern and a northern counterpart which differ in species composition. The forest groups in themselves are highly diverse, for example the group $5^{\text {th }}$ in Table 4, has 5 subgroups and 23 categories (Table 5). Of the 16 forest groups, the tropical deciduous accounts for $38.2 \%$ of the total forest area (Fig. 3). The distribution of woody species in this forest group is determined by small-scale variations in environmental variables resulting into patchiness in communities (Chaturvedi et al., 2011a; Chaturvedi and Raghubanshi, 2014; Chaturvedi and Raghubanshi, 2015). Due to the patchy distribution of tree assemblages, tropical dry forests generally show uneven distribution of aboveground tree biomass, carbon density and carbon accumulation (Chaturvedi et al., 2011b; Chaturvedi et al., 2011c; Chaturvedi et al., 2012; Chaturvedi et al., 2017a). The deciduous forest is species poor but has been shown to be a mosaic of communities with distinct species composition; each of these communities is distributed in non-contiguous patches leading to immense diversity (Jha and Singh, 1990). A detrended

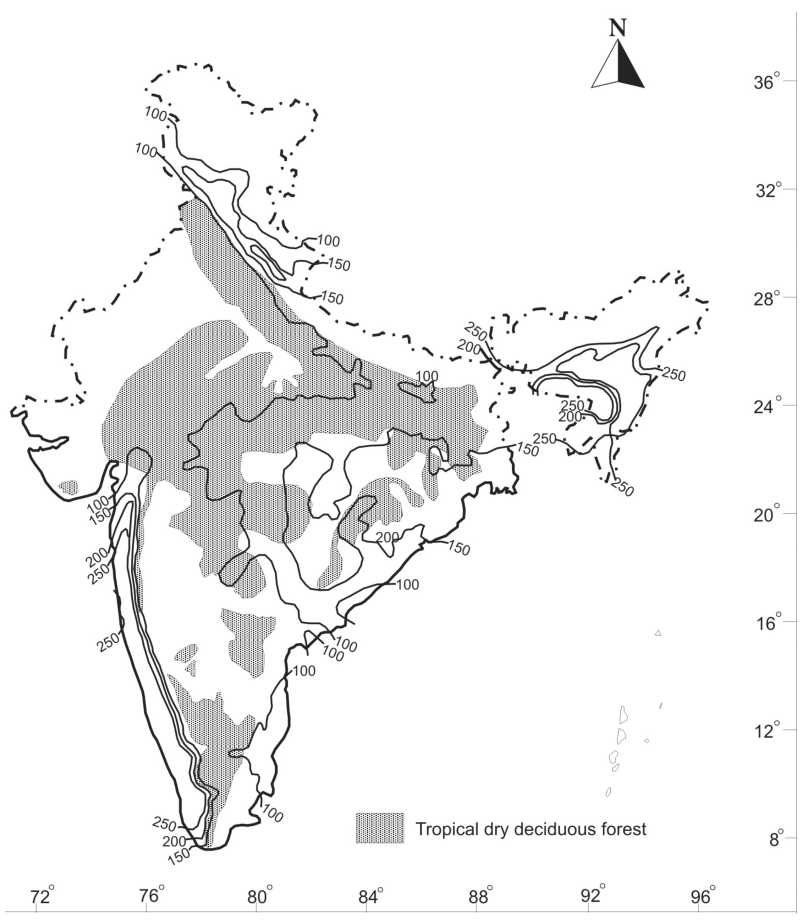

Fig. 3: Potential distribution of tropical dry deciduous forest in India. The contour lines represent annual rainfall in centimetres (Source: Singh and Singh, 2011)

\section{Legend (Biome) \\ Tropical Rain Forest
Troplcal Rain Forest (Lower Mortane) Troplcal Raln Forest (Plalns) Troplcal Wet Forest Troplcal Wet Forest (Lower Montane) Tropical Molst Forest Troplcal Molst Forest (Sub Alpine) Troplcal Molst Forest (Lower Montane) Troplcal Molst Forest(Plalns) Troplcal Dry Forest Tropical Dry Forest (Montane) Tropical Dry Forest (Lower Montane) Tropical Very Dry Forest (Plains) Tropical Dry Scrub (Plains) Troplcal Thom Woodland Tropical Thom Woodland (Lower Montane) Tropical Thom Woodland(Plains) Tropical Desert Scrub (Lower Montane) Tropical Desert (Plains) Himalayan Temperate Tundra Dry Tundra (Alpine) Very Dry Tundra (Alwar)}


Table 2. Important floral groups of India (Adapted from MoEF, 2009)

\begin{tabular}{|c|c|c|c|}
\hline Floral groups & $\begin{array}{l}\text { Species/Genera/ } \\
\text { Families }\end{array}$ & $\begin{array}{l}\text { Important Families } \\
\text { (no. of species) }\end{array}$ & Remarks \\
\hline Angiosperms & $\begin{array}{l}\text { about } 17,527 \text { species } \\
\text { in } 2,984 \text { genera and } \\
247 \text { families }\end{array}$ & $\begin{array}{l}\text { Poaceae (1291); Orchidaceae } \\
\text { (1229); Leguminosae (1225); } \\
\text { Asteraceae (892); Rubiaceae } \\
\text { (616); Cyperaceae (545); } \\
\text { Euphorbiaceae (527); } \\
\text { Acanthaceae (510) }\end{array}$ & $\begin{array}{l}\text { India contains more than } 7 \% \text { of the world's known flowering } \\
\text { plants; about } 4,900 \text { species are endemic to the country; } \\
\text { Labiatae and Compositae are more abundant in the temperate } \\
\text { regions while the rest are largely tropical in distribution }\end{array}$ \\
\hline Gymnosperms & $\begin{array}{l}\text { about } 67 \text { species in } \\
20 \text { genera }\end{array}$ & $\begin{array}{l}\text { Pinaceae (15); Cupressaceae } \\
\text { (13); Ephedraceae (7); } \\
\text { Gnetaceae (5) }\end{array}$ & $\begin{array}{l}\text { species of Gnetum and Cycas are mostly confined to North } \\
\text { Eastern region, Eastern and Western Ghats, and Andaman } \\
\text { and Nicobar Islands }\end{array}$ \\
\hline Pteridophytes & $\begin{array}{l}\text { about } 1200 \text { species } \\
\text { in } 204 \text { genera }\end{array}$ & $\begin{array}{l}\text { Polypodiaceae (137); } \\
\text { Dryopteridaceae }(125) ; \\
\text { Athyriaceae (97); Thelypteri- } \\
\text { daceae (83); Selaginellaceae } \\
(62)\end{array}$ & $\begin{array}{l}\text { about } 17 \% \text { of the species are endemic to India; north-eastern } \\
\text { region (including Eastern Himalaya) contains about } 845 \text { species, } \\
\text { followed by South India (including Eastern and Western Ghats) } \\
\text { with } 345 \text { species and North India (including Western } \\
\text { Himalaya) with } 340 \text { species; dominant genera are Selaginella } \\
\text { ( } 62 \text { species), Asplenium ( } 45 \text { species) and Polystichum ( } 45 \\
\text { species) }\end{array}$ \\
\hline Bryophytes & $\begin{array}{l}\text { about } 2500 \text { species } \\
\text { in } 482 \text { genera and } \\
106 \text { families }\end{array}$ & $\begin{array}{l}\text { Lejeuneaceae (155); Pottiaceae } \\
\text { (129); Dicranaceae (119); } \\
\text { Bryaceae (98); Sematophy- } \\
\text { llaceae (92) }\end{array}$ & $\begin{array}{l}\text { second largest group of green plants in India distributed } \\
\text { largely in Eastern Himalaya, North-eastern India, Western } \\
\text { Himalaya and the Western Ghats; } 19 \text { genera and } 629 \\
\text { species are endemic to India; major component include mosses } \\
\text { (1576 species), and liverworts and hornworts ( } 924 \text { species) }\end{array}$ \\
\hline Lichens & $\begin{array}{l}\text { about } 2,223 \text { species in } \\
283 \text { genera and } \\
72 \text { families }\end{array}$ & $\begin{array}{l}\text { Parmeliaceae; Graphidaceae; } \\
\text { Physciaceae; Usneaceae; } \\
\text { Cladoniaceae }\end{array}$ & $\begin{array}{l}\text { Western Ghats are the richest region with } 800 \text { species } \\
(38 \%) \text { followed by Eastern Himalaya with } 759 \text { species }(37 \%) \\
\text { and Western Himalaya with } 550 \text { species ( } 27 \%)\end{array}$ \\
\hline Fungi & $\begin{array}{l}\text { about } 14,500 \text { species } \\
\text { in } 2,300 \text { genera and } \\
250 \text { families }\end{array}$ & $\begin{array}{l}\text { Deuteromycetes }(6000) \\
\text { Ascomycetes }(3500) \\
\text { Basidiomycetes }(3400)\end{array}$ & $\begin{array}{l}\text { maximum diversity in the Western Ghats followed by the } \\
\text { Eastern Himalaya and the Western Himalaya; about } 3500 \\
\text { species are endemic to the country; dominant genera are } \\
\text { Cercospora ( } 707 \text { species); Puccinia ( } 328 \text { species); Phyllosticta } \\
\text { (280 species) }\end{array}$ \\
\hline Algae & $\begin{array}{l}\text { about } 7,175 \text { species } \\
\text { in } 666 \text { genera }\end{array}$ & $\begin{array}{l}\text { Chlorophyceae }(4,495) \\
\text { Cyanophyceae }(1,453) \\
\text { Bacillariophyceae }(516)\end{array}$ & $\begin{array}{l}\text { found in a variety of habitats ranging from aquatic } \\
\text { (both fresh water and marine) to terrestrial }\end{array}$ \\
\hline
\end{tabular}

correspondence analysis (DCA) of 48 stands of a dry tropical forest indicated the presence of five distinct communities (Figure 4) which were associated with soil texture. Plant species in these forests have important functional traits that enable acquisition of limiting nutrients and water (Chaturvedi et al., 2011b; Chaturvedi et al., 2011d; Chaturvedi et al., 2013; Chaturvedi et al., 2014; Chaturvedi and Raghubanshi, 2013; Chaturvedi and Raghubanshi, 2016). The other predominant forest group is the moist deciduous (Group-3) covering $30.3 \%$ of the forest area of the country, having 4 sub-groups and 15 categories (Table 4).

Tropical forests of the country are divided into a series of successively drier climatic types. Among the six major forest groups, the dense and multilayered moist tropical forests occur in the areas of high temperatures and rainfall and comprise (i) wetevergreen forests, characterized by dense growth of tall trees, and rich in climbers, lianas, epiphytes and shrubs but poor in herbs and grasses; (ii) semievergreen forests characterized by dense growth of intermixed deciduous and evergreen trees and a rich carpet layer of herbs, grasses and ferns; (iii) moistdeciduous forests, characterized by the dominance of deciduous trees with a lower storey of evergreen trees and shrubs; (iv) littoral and swamp forests characterized by the dominance of halophytic evergreen plants.

Dry tropical forests comprise (i) dry-deciduous 
Table 3: Important wild fauna of India. (Adapted from Singh et al., 2015).

\begin{tabular}{lll}
\hline S.No. & Biogeographic zone & Important wild fauna \\
\hline 1. & Trans-Himalayas & $\begin{array}{l}\text { Wild Yak, Ass, Gazelle, Four-Horned Antelope, Snow Leopard, Tibetan Wolf, Lesser Cat (Pallas' } \\
\text { Cat), Fox, Marbled Pole Cat, Royals Pika,Himalayan Marmot, Black Necked Crane }\end{array}$ \\
2. & Himalaya & $\begin{array}{l}\text { Tibetan Ass, Sikkim Stag, Musk Deer, Tahr, Beharal, Ibex, Mishmi Takin, Sun Bear,Gibbon, Binturong, } \\
\text { Red Pandas, Lesser Cats, Jungle Fowl, Markhor, Serow }\end{array}$ \\
3. & Desert & Wild Ass, Desert Fox, Indian Desert Cat, Honbara Bustard, Sand Grouse, Chinkara, Blackbuck, \\
& Wolf, Caracal, Great Indian Bustard, Flamingoes
\end{tabular}

forest, characterized by abundance of shrubs and open canopy of small trees, which experience about six months of dry period in the annual cycle; (ii) thorn forests which experience more than six months of dry period each year and are characterized by the sparse distribution of small, mostly thorny trees with abundance of shrubs. Grasses and herbs appear during the brief rainy season when trees and shrubs also develop leaves; (iii) dry-evergreen forests experiencing high temperature and small rainfall available during the summer season. Bamboos are absent from these forests but grasses and small trees are abundant.

Montane sub-tropical forests are present between the altitudes of $1000 \mathrm{~m}$ and $2000 \mathrm{~m}$ and experience cooler climate than tropical but warmer than temperate forests. Semi-xerophytic evergreen plants are predominantly present and the forests are categorized into (i) broad-leaved hill forests, characterized by abundance of climbers and epiphytic ferns and orchids, and dense growth of evergreen broad-leaved trees; (ii) pine forests, comprising the open formations of pine trees; (iii) dry-evergreen forests, mainly characterized by the presence of smallleaved evergreen plants and thorny xerophytes.
Montane temperate forests are found in areas having low temperature with comparatively high humidity. The forests comprise tall conifers or angiospermic evergreen trees laden with epiphytic mosses, lichens and ferns. These forests are categorized into three groups: (i) montane wet temperate forests which have a northern and a southern category. The northern category (Eastern Himalaya) is characterized by dense evergreen or semi-evergreen broad-leaved trees of up to $25 \mathrm{~m}$ height. In the southern category, trees are relatively

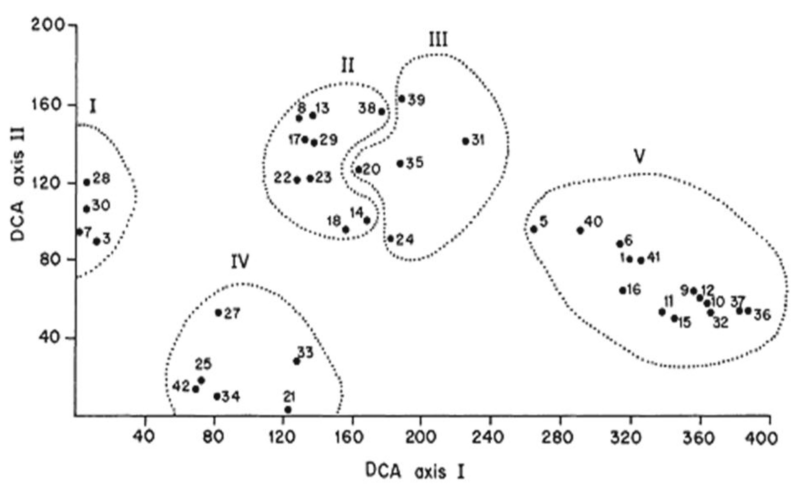

Fig. 4: DCA ordination of dry tropical forest stands (Source: Jha and Singh, 1990) 
Table 4: Diversity of forest ecosystems: forest types, their dominant species, and distribution (based on Champion and Seth, 1968)

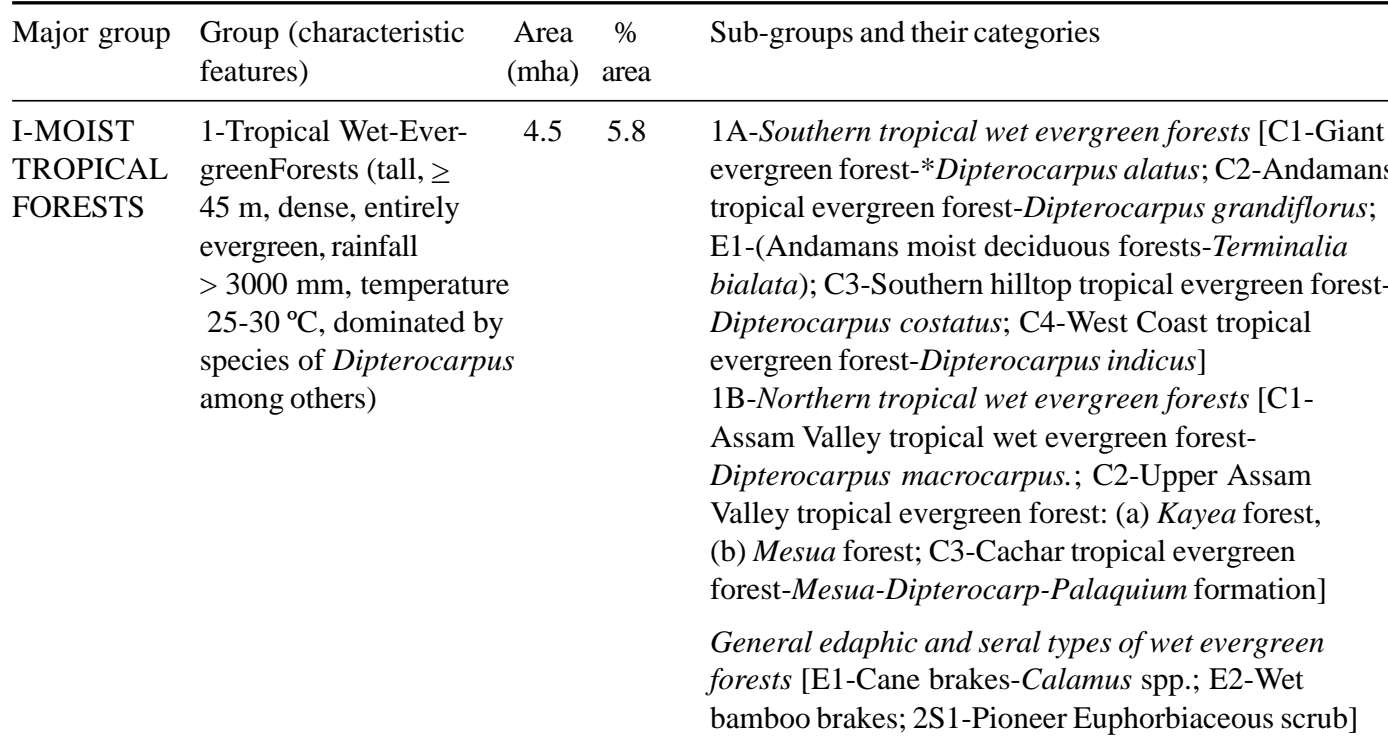

2-Tropical Semi-Ever- $\quad 1.9 \quad 2.5$ green Forests (dominants are deciduous, but evergreens predominate in lower or middle canopy, may reach $40 \mathrm{~m}$ height, rainfall $2000-2500 \mathrm{~mm}$, temperature $25-32{ }^{\circ} \mathrm{C}$, dominated by Magnolia, Cinnamomum, Terminalia, Xylia, etc.).
3-Tropical MoistDeciduous Forests (dominants deciduous, lower storey trees usually evergreen, may reach $40 \mathrm{~m}$ height,
$23.3 \quad 30.3 \quad 3 \mathrm{~A}$-Andamans moist deciduous forests [C1-Andamans Andaman Islands, moist deciduous forest-Pterocarpus dalbergioides; 2S1- Nicobars

Andamans secondary moist deciduous forest-Canarium euphyllum] 3B-South Indian moist deciduous forests [C1-Moist Maharashtra, teak-bearing forest: (1a) Very moist teak forest-Termi- Madhya Pradesh, 
rainfall $1200-2500 \mathrm{~mm}$, temperature $25-30{ }^{\circ} \mathrm{C}$, may or may not bear Tectona grandis, or Shorea robusta)

\author{
4-Littoral and Swamp $\quad 0.7$
Forests (mainly evergreen, \\ include mangrove and \\ freshwater swamp \\ forests, dominated by \\ Rhizophora, Bruguiera, \\ Ipomea, Phoenix, \\ Barringtonia, etc)
}

nalia crenulata, (1b) Moist teak forest-Tectona grandis, Gujarat, Mysore, (1c) Slightly moist teak forest-Tectona grandis; Madras, Kerala C2-Southern moist mixed deciduous forestPterocarpus marsupium; 2S1-Southern secondary moist mixed deciduous forest-Terminalia paniculata]

3C-North Indian moist deciduous forests [C1-Very Uttrakhand, moist sal-bearing forest: (1a) Eastern hill sal forest Uttar Pradesh, \{(i) East Himalayan sal, (ii) Khasi hill sal\}, (1b) Eastern Bihar, Orissa, bhabar sal forest \{(i) East Himalayan upper bhabar West Bengal, sal, (ii) East Himalayan lower bhabar sal\}, (1c) Eastern Assam, tarai sal forest, (1d) Peninsular (coastal) sal forest; C2- Madhya Pradesh Moist sal-bearing forest: (2a) Moist Siwalik sal forest, (2b) Moist bhabar sal forest \{(i) Bhabar-dun sal, (ii) Damar sal\}, (2c) Moist tarai sal forest, (2d) Moist plains sal forest \{(i) Western light alluvium plains sal, (ii) App. Chandar sal, (iii) Eastern heavy alluvium plains sal, (iv) App. Kamrup sal\}, (2e) Moist peninsular sal forest \{(i) Moist peninsular high level sal, (ii) Moist peninsular low level sal, (iii) Moist peninsular valley sal \}; DS1-Moist sal savannah; C3-Moist mixed deciduous forest (without sal): (3a) West Gangetic moist mixed deciduous forestAlbizzia procera, (3b) East Himalayan moist mixed deciduous forest-Lagerstroemia parviflora; 2S1-Northern secondary moist mixed deciduous forest-Mangifera indica; 2S2-(Secondary Euphorbiaceous scrub)-Terminalia tomentosa]

General edaphic and seral types of moist deciduous forests [E1-Terminalia tomentosa forest; 1S1-Low alluvial savannah woodland (Salmalia-Albizzia); 1S2Eastern hollock forests (Terminalia myriocarpa): (2a) Terminalia-Lagerstroemia, (2b) TerminaliaDuabanga]; 2S1-(Dry bamboo brakes)Dendrocalamus strictus]

4A-Littoral forests [L1-Littoral forest-Manilkara littoralis]

Uttar Pradesh, Bihar, West Bengal, Assam, Madhya Pradesh

Andaman and Nicobar Islands 4B-Tidal swamp forests [TS1-Mangrove scrub-Ceriops Delta of Ganges roxburghiana; TS2-Mangrove forest-Rhizophora and Brahmaputra candelaria; TS3-Saltwater mixed forest-Heritiera minor; rivers TS4-Brakish water mixed forest-Heritiera minor; E1-Palm swamp-Phoenix paludosa] 4C-Tropical freshwater swamp forests [FS1-Myristica swamp forest-Myristica magnifica; FS2-Submontane hill valley swamp forest-Eugenia spp.; FS3-Creeper swamp forest-Magnolia griffithii]

4D-Tropical seasonal swamp forests [SS1-Eastern seasonal swamp forest-Altingia excelsa; SS2-

Barringtonia swamp forest- Barringtonia acutangula; SS3-Syzygium cumini swamp low forest; SS4-Eastern seasonal swamp low forest-Cephalanthus occidentalis; SS5-Eastern Dillenia swamp forest-Dillenia indica; 2S1-(Syzygium parkland); 2S2-(Eastern wet alluvial grassland)-Vetiveria zizanioides]

4E-Tropical riparian fringing forests [RS1-Riparian fringing forest-Terminalia arjuna ]

Banks of bigger rivers

Kerala, Uttrakhand, Brahmaputra Valley

Brahmaputra Valley, Uttar Pradesh,

Assam, West Bengal

II-DRY 5-Tropical Dry$29.4 \quad 38.2$
Maharashtra, Madhya Pradesh, 


\begin{tabular}{|c|c|c|c|c|c|}
\hline \multirow[t]{6}{*}{ FORESTS } & \multirow[t]{3}{*}{$\begin{array}{l}\text { (entirely deciduous } \\
\text { or nearly so, rainfall } \\
800-1200 \mathrm{~mm} \text {, temp. } \\
25-32^{\circ} \mathrm{C} \text {, some forests } \\
\text { bear Tectona grandis } \\
\text { or Shorea robusta, } \\
\text { others are devoid of } \\
\text { these species and have } \\
\text { species of Anogeissus } \\
\text { and Terminalia, etc.). }\end{array}$} & & & $\begin{array}{l}\text { teak forest; C2-Dry red sanders-bearing forest- } \\
\text { Pterocarpus santalinus; C3-Southern dry mixed } \\
\text { deciduous forest-Terminalia tomentosa] } \\
\text { 5B-Northern tropical dry deciduous forests [C1-Dry } \\
\text { sal bearing forest: (1a) Dry Siwalik sal forest, (1b) Dry } \\
\text { plains sal forest, (1c) Dry peninsular sal forest; } \\
\text { C2-Northern dry mixed deciduous forest-Acacia } \\
\text { catechu] } \\
\text { Degradation stages of tropical dry deciduous forests } \\
\text { [DS1-Dry deciduous scrub-Nyctanthes arbortristis; } \\
\text { DS2-Dry savannah forest-Gardenia turgida; DS3- } \\
\text { (Euphorbia scrub)-Euphorbia neriifolia; DS4- } \\
\text { (Dry grassland)-Sehima nervosum] }\end{array}$ & $\begin{array}{l}\text { Andhra Pradesh, } \\
\text { Madras, Mysore } \\
\text { Punjab, } \\
\text { Uttar Pradesh, } \\
\text { Bihar, Orissa, } \\
\text { West Bengal } \\
\text { Maharashtra, } \\
\text { Madhya Pradesh, } \\
\text { Andhra Pradesh, } \\
\text { Madras, Mysore, } \\
\text { Punjab, Uttar } \\
\text { Pradesh, Bihar, } \\
\text { Orissa, West Bengal }\end{array}$ \\
\hline & & & & $\begin{array}{l}\text { General edaphic types of dry deciduous forests [E1- } \\
\text { Anogeissus pendula forest; DS1-Anogeissus pendula } \\
\text { scrub; E2-Boswellia forest-Boswellia serrata; E3- } \\
\text { Babul forest-Acacia arabica; E4-Hardwickia forest- } \\
\text { Hardwickia binata; E5-Butea forest-Butea monosperma; } \\
\text { E6-Aegle forest- Aegle marmelos; E7-Laterite thorn } \\
\text { forest-Gardenia spp.; E8-Saline/alkaline scrub savannah: } \\
\text { (8a) Phoenix savannah-Phoenix sylvestris, (8b) Babul } \\
\text { savannah-Acacia arabica, (8c) Salvadora-Tamarix scrub } \\
\text { E9-Dry bamboo brake-Dendrocalamus strictus] }\end{array}$ & $\begin{array}{l}\text { Uttar Pradesh, } \\
\text { Rajasthan, Madhya } \\
\text { Pradesh, } \\
\text { Maharashtra, } \\
\text {; Andhra Pradesh, } \\
\text { Punjab, Bihar, } \\
\text { : Kerala, Gujarat, } \\
\text { Mysore, Madras }\end{array}$ \\
\hline & & & & $\begin{array}{l}\text { General seral types of dry deciduous forests [1S1-Dry } \\
\text { tropical riverine forest-Terminalia arjuna; } 1 \mathrm{~S} 2-\text { Khair- } \\
\text { sissu forest-Acacia catechu; } 1 \mathrm{~S} 3 \text {-Inundation babul } \\
\text { forest-Acacia arabica; } 2 \mathrm{~S} 1-\mathrm{Secondary} \text { dry deciduous } \\
\text { forest-Prosopis spicigera] }\end{array}$ & $\begin{array}{l}\text { Rajasthan, } \\
\text { Punjab, Uttar } \\
\text { Pradesh, Bihar, } \\
\text { West Bengal, } \\
\text { Assam }\end{array}$ \\
\hline & \multirow[t]{2}{*}{$\begin{array}{l}\text { 6-Tropical Thorn } \\
\text { Forests (deciduous } \\
\text { with low thorny trees, } \\
\text { rainfall } 200-800 \mathrm{~mm}, \\
\text { temperature } 27-30^{\circ} \mathrm{C}, \\
\text { Acacia, Balanites, } \\
\text { Prosopis, Salvadora, } \\
\text { etc., predominate) }\end{array}$} & \multirow[t]{2}{*}{5.2} & \multirow[t]{2}{*}{6.7} & $\begin{array}{l}\text { 6A-Southern tropical thorn forests [C1-Southern thorn } \\
\text { forest-Acacia leucophloea; C2-Karnatak umbrella thorn } \\
\text { forest-Acacia planifrons; DS1-Southern thorn scrub- } \\
\text { Zizyphus xylopyrus; DS2-Southern Euphorbia scrub- } \\
\text { Euphorbia tirucalli] } \\
\text { 6B-Northern tropical thorn forests [C1-Desert thorn } \\
\text { forest-Prosopis spicigera; C2-Ravine thorn forest- } \\
\text { Acacia leucophloea; DS1-Zizyphus scrub-Acacia } \\
\text { leucophloea; DS2-Tropical Euphorbia scrub-Euphorbia } \\
\text { neriifolia] }\end{array}$ & $\begin{array}{l}\text { Punjab, Uttar } \\
\text { Pradesh, Madhya } \\
\text { Pradesh, Gujarat, } \\
\text { Rajasthan }\end{array}$ \\
\hline & & & & $\begin{array}{l}\text { General edaphic, degraded and seral types of moist } \\
\text { deciduous forests [E1-(Euphorbia } \text { scrub)-Euphorbia } \\
\text { neriifolia; E2-Acacia senegal forest; E3-Rann saline } \\
\text { thorn forest-Prosopis juliflora; E4-Salvadora scrub- } \\
\text { Salvadora oleoides; DS1-Cassia auriculata scrub; } \\
\text { 1S1-Desert dune scrub-Prosopis spicigera] }\end{array}$ & $\begin{array}{l}\text { Rajasthan, } \\
\text { Gujarat, Punjab }\end{array}$ \\
\hline & $\begin{array}{l}\text { 7-Tropical Dry-Ever- } \\
\text { green Forests (hard- } \\
\text { leaved evergreen trees, } \\
\text { rainfall } 870-1200 \mathrm{~mm}, \\
\text { dominated by Manilkara, } \\
\text { and Memecylon, etc.) }\end{array}$ & 0.1 & 0.1 & $\begin{array}{l}\text { [C1-Tropical dry evergreen forest-Manilkara hexandra; } \\
\text { DS1- Tropical dry evergreen scrub-Memecylon edule] }\end{array}$ & $\begin{array}{l}\text { Andhra Pradesh, } \\
\text { Madras }\end{array}$ \\
\hline $\begin{array}{l}\text { III-MON- } \\
\text { TANE SUB- } \\
\text { TROPICAL } \\
\text { FORESTS }\end{array}$ & $\begin{array}{l}8 \text {-Sub-Tropical Broad } \\
\text { Leaved Hill Forests } \\
\text { (largely evergreen, } \\
\text { rainfall } 1000-3000 \mathrm{~mm} \\
\text { (Cherrapunji, } 11000 \mathrm{~mm} \text { ), }\end{array}$ & 0.3 & 0.4 & $\begin{array}{l}\text { 8A-Southern subtropical broadleaved hill forests [C1- } \\
\text { Nilgiri subtropical hill forest-Calophyllum elatum; DS1- } \\
\text { South Indian sub-tropical hill savannah (woodland)- } \\
\text { Dalbergia latifolia; E1-Reed brakes-Ochlandra wightii; } \\
\text { C2-Western subtropical hill forest-Syzygium cumini; }\end{array}$ & $\begin{array}{l}\text { Kerala, Madras, } \\
\text { Mysore, Mumbai, } \\
\text { Rajasthan, } \\
\text { Madhya Pradesh, } \\
\text { Bihar, Orissa }\end{array}$ \\
\hline
\end{tabular}


in southern India species of Calophyllum, Rhododendron niligiricum, etc. and in eastern Himalaya, species of Quercus predominate)

9-Sub-Tropical Pine $3.7 \quad 5.0$

Forests (pine associations predominate in low altitudes of Himalaya, rainfall 1000-3000mm, Pinus roxburghii (western Himalaya), P. kesiya (eastern Himalaya)

10-Sub-Tropical Dry- $0.2 \quad 0.2$ Evergreen Forests (low xerophytic forest and scrub in the foothills of western Himalaya, Olea and Acacia spp.)

IV-MON-

TANE

11-Montane Wet

Temperate Forests

TEMPE-

RATE (closed evergreen high FORESTS forests, rainfall 1500-> $5000 \mathrm{~mm}$, in southern India shola forests (Ternstroemia, Ilex, Rhododendron), in eastern Himalaya Quercus forests with Machilus, Acer, Rhododendron, etc.)

12-Himalayan Moist Temperate Forests (all along Himalaya (1500-3300m altitude), rainfall $1000-2500 \mathrm{~mm}$, several conifers (e.g., Abies, Cedrus, Picea, Tsuga) and broadleaved species (Quercus, Rhododendron, Acer, Ulmex, etc.), composition differs with altitude and location (western vs. eastern Himalaya)
C3-Central Indian subtropical hill forest-Carallia brachiata; DS-(Degradation stages of southern subtropical broad-leaved hill forests)] 8B-Northern subtropical broadleaved wet hill forests [C1-East Himalayan subtropical wet hill forestCastanopsis tribuloides; C2-Khasi subtropical wet hill forest-Quercus spp.; 2S1-(Assam subtropical pine forest); DS1-(Assam subtropical hill savannah woodland)]

[C1-Himalayan subtropical pine forest: (1a) Lower or Siwalik chir pine forest-Pinus roxburghii, (1b)

Punjab, Uttrakhand, Upper or Himalayan chir pine forest-Pinus roxburghii; Assam, Manipur DS1-Himalayan subtropical scrub-Carissa spp.; DS2-Subtropical Euphorbia scrub-Euphorbia royleana; C2-Assam subtropical pine forests-Pinus insularis; DS1-Assam subtropical pine savannah]

[C1-Subtropical dry evergreen forest: (1a) Olea cuspidata scrub forest, (1b) Acacia modesta scrub forest; DS1-Dodonaea scrub-Dodonaea viscosa]

Jammu, Punjab

11A-Southern montane wet temperate forests [C1Madras, Kerala Southern montane wet temperate forest-Ternstroemia gymnanthera; DS1-Southern montane wet scrubHypericum mysorense; DS2- Southern montane wet grassland-Bothriochloa pertusa ]

11B-Northern montane wet temperate forests [C1East Himalayan wet temperate forests: (1a) Lauraceous Assam, Manipur, forest-Machilus edulis, (1b) Buk oak forest-Quercus lamellosa, (1c) High-level oak forest-Quercus pachyphylla; C2-Naga hills wet temperate forestsMagnolia spp.]

[C1-Lower Western Himalayan temperate forest: (1a) Ban oak forest (Quercus incana), (1b) Moru oak forest (Quercus dilatata); DS1-Oak scrub: (1c) Moist deodar forest-Cedrus deodara, (1d) Western mixed coniferous forest (spruce, blue pine, silver fir)-Picea sp., West Bengal, Cedrus spp., Abies pindrow, (1e) Moist temperate Assam deciduous forest-Aesculus indica, (1f) (Low-level blue pine forest (Pinus wallichiana)); DS1-Oak scrub-Q. incana; DS2-Himalayan temperate secondary scrub-Plectranthus rugosus; C2-Upper West Himalayan temperate forest: (2a) Kharsu oak forest (Quercus semecarpifolia), (2b) West Himalayan upper oak/fir forest-Abies pindrow, (2c) (Moist temperate deciduous forest)-Aesculus indica; C3-East Himalayan moist temperate forest:

(3a) East Himalayan mixed coniferous forestTsuga dumosa, (3b) Abies delavayi forest]

Degradation stages of Himalayan moist temperate forests [DS1-Montane bamboo brakes-Arundinaria spp.; DS2-Himalayan temperate parkland-Prunus padus; DS3- Himalayan temperate pastures-Festuca spp.]

General edaphic and seral types of Himalayan moist Uttrakhand, temperate forests [E1-Cypress forest-Cypressus
Kashmir, Punjab, Himachal Pradesh, Uttrakhand, Assam Western to Eastern Himalayas Punjab, Jammu
Jammu and 


\begin{tabular}{|c|c|c|c|c|c|}
\hline & \multirow{4}{*}{$\begin{array}{l}\text { 13-Himalayan Dry } \\
\text { Temperate Forests } \\
\text { (essentially open } \\
\text { formations, along inner } \\
\text { valleys of Himalaya, } \\
\text { rainfall < } 1000 \mathrm{~mm} \text {, } \\
\text { conifers like } \text { Pinus } \\
\text { (e.g., P. gerardiana), } \\
\text { Picea and Juniperus, } \\
\text { predominate) }\end{array}$} & \multirow[b]{2}{*}{0.2} & \multirow[b]{2}{*}{0.2} & \multicolumn{2}{|l|}{$\begin{array}{l}\text { torulosa; } 1 \mathrm{~S} 1 \text {-Alder forest-Alnus nitida; } 1 \mathrm{~S} 2 \text {-Riverain } \\
\text { blue pine forest-Pinus wallichiana; } 2 \mathrm{~S} 1 \text {-Low-level } \\
\text { blue pine forest- Pinus wallichiana }]\end{array}$} \\
\hline & & & & $\begin{array}{l}\text { (i) Western types [C1-Dry broadleaved and coniferous } \\
\text { forest (Quercus ilex-Pinus gerardiana); C2-Dry } \\
\text { temperate coniferous forest: (2a) Neoza pine forest } \\
\text { (Pinus gerardiana), (2b) Dry deodar forest-Cedrus } \\
\text { deodara; DS1-Pohu scrub-Parrotia jacquemontiana; } \\
\text { DS2-Dry temperate scrub-Plectranthus rugosus; } \\
\text { C3-(West Himalayan dry temperate deciduous forest)- } \\
\text { Corylus colurna; C4-West Himalayan high-level dry blue } \\
\text { pine forest-Pinus wallichiana; C5-West Himalayan } \\
\text { dry juniper forest (Juniperus macropoda)] }\end{array}$ & $\begin{array}{l}\text { Jammu and } \\
\text { Kashmir, } \\
\text { Punjab, Himachal } \\
\text { Pradesh, Uttrakhand }\end{array}$ \\
\hline & & & & $\begin{array}{l}\text { (ii) Eastern types [C6-East Himalayan dry temperate } \\
\text { coniferous forest-Picea spinulosa; E1-Larch forest- } \\
\text { Larix griffithiana; C7-East Himalayan dry juniper/ } \\
\text { birch forest-Juniperus wallichiana] }\end{array}$ & Sikkim \\
\hline & & & & $\begin{array}{l}\text { General seral types of dry temperate forests [1S1- } \\
\text { Hippophae/Myricaria scrub-Hippophae salicifolia; } \\
\text { 1S2-Populus/Salix forest-Populus euphratica; } \\
\text { 1S3-(Western high-level dry blue pine forest)-Pinus } \\
\text { wallichiana] }\end{array}$ & $\begin{array}{l}\text { Western to Eastern } \\
\text { Himalayas }\end{array}$ \\
\hline $\begin{array}{l}\text { V-SUB- } \\
\text { ALPINE } \\
\text { FORESTS }\end{array}$ & $\begin{array}{l}\text { 14-Sub-Alpine Forests } \\
\text { (stunted deciduous or } \\
\text { evergreen forests with } \\
\text { conifers (2900-3500m } \\
\text { altitude), mean annual } \\
\text { temp. } 2^{\circ} \mathrm{C} \text {, rainfall } \\
83-600 \mathrm{~mm} \text {, species } \\
\text { of Abies, Betula and } \\
\text { Rhododendron predomina }\end{array}$ & - & - & $\begin{array}{l}\text { [C1-West-Himalayan sub-alpine birch/fir forests } \\
\text { (Betula/Abies): (1a) West Himalayan sub-alpine fir } \\
\text { forest-Abies spectabilis, (1b) West Himalayan sub- } \\
\text { alpine birch/fir forest-Betula utilis; C2-East Himalayan } \\
\text { sub-alpine birch/fir forest-Abies densa] } \\
\text { Seral and degraded types of sub-alpine forests [1S1- } \\
\text { (Hippophae/Myricaria brakes)-Hippophae salicifolia; } \\
\text { 1S2-(Deciduous sub-alpine scrub)-Syringa emodi; } \\
\text { 2S1-(Sub-alpine blue pine-Pinus wallichiana forest); } \\
\text { DS1-Sub-alpine pastures-Agropyron longearistatum] }\end{array}$ & $\begin{array}{l}\text { Jammu and } \\
\text { Kashmir, } \\
\text { Punjab, Himachal } \\
\text { Pradesh, Uttrakhand, } \\
\text { Bengal, Assam, } \\
\text { Manipur } \\
\text { Jammu \& Kashmir, } \\
\text { Punjab, Himachal } \\
\text { Pradesh, Uttrakhand, } \\
\text { Bengal, Assam, } \\
\text { Manipur }\end{array}$ \\
\hline \multirow[t]{2}{*}{$\begin{array}{l}\text { VI-ALPINE } \\
\text { SCRUB }\end{array}$} & $\begin{array}{l}\text { 15-Moist-Alpine Scrub } \\
\text { (throughout Himalaya, } \\
\text { above timber line to } \\
5500 \mathrm{~m} \text { altitude, species } \\
\text { of Juniperus, Rhodo- } \\
\text { dendron predominate) }\end{array}$ & 3.3 & 4.3 & $\begin{array}{l}\text { [C1-Birch/Rhododendron scrub forest-Betula utilis; } \\
\text { C2-Deciduous alpine scrub-Syringa emodi; E1-Dwarf } \\
\text { Rhododendron scrub- Rhododendron campanulatum; } \\
\text { E2-(Dwarf juniper scrub)-Juniperus communis; } \\
\text { C3-(Alpine pastures)-Primula } \text { spp.] }\end{array}$ & $\begin{array}{l}\text { Jammu \& Kashmir, } \\
\text { Uttrakhand, } \\
\text { Sikkim, Manipur }\end{array}$ \\
\hline & $\begin{array}{l}\text { 16-Dry-Alpine Scrub } \\
\text { (in low rainfall areas, } \\
\text { upto } 5500 \mathrm{~m} \text { altitude, } \\
\text { as xerophytic, e.g., } \\
\text { Juniperus, Eurotia, } \\
\text { Salix, etc.) }\end{array}$ & - & - & $\begin{array}{l}\text { [C1-Dry alpine scrub-Eurotia ceratoides; E1-Dwarf } \\
\text { juniper scrub-Juniperus communis] }\end{array}$ & $\begin{array}{l}\text { Uttrakhand, Punjab, } \\
\text { Himachal Pradesh, } \\
\text { Jammu \& Kashmir, }\end{array}$ \\
\hline
\end{tabular}

*Dominant species

smaller, broad-leaved with dense leaf canopy, abundant epiphytic flora and rich herbaceous undergrowth; Shola forests belong to this category, (ii) Himalayan moist forests comprise tall (up to $45 \mathrm{~m}$ high) conifers, oaks or their mixture with thin partly deciduous undergrowth; (iii) Himalayan dry forests are essentially open formations and occur in the inner ranges of Himalaya with very low rainfall. These forests are characterized by the dominance of evergreen oaks and conifers. Undergrowth is formed by scrubs. The group has a western (characteristic species Pinus gerardiana) and an eastern counterpart (characteristic species Picea spinulosa); both types have an abundance of Junipers.

Sub-alpine forests occur in the Himalaya and 
Table 5: Tropical dry deciduous forest categories of India (based on Champion and Seth, 1968).

\begin{tabular}{|c|c|c|c|}
\hline \multicolumn{2}{|c|}{ Southern tropical dry deciduous forests } & \multicolumn{2}{|c|}{ Northern tropical dry deciduous forests } \\
\hline \multicolumn{2}{|l|}{ Dry teak bearing forest } & \multicolumn{2}{|l|}{ Dry sal-bearing forest } \\
\hline \multicolumn{2}{|l|}{ Dry teak forest } & \multicolumn{2}{|l|}{ Dry Siwalik sal forest } \\
\hline \multicolumn{2}{|l|}{ Very dry teak forest } & \multicolumn{2}{|l|}{ Dry plains sal forest } \\
\hline \multicolumn{2}{|c|}{ Dry red sanders bearing forest } & \multicolumn{2}{|c|}{ Dry peninsular sal forest } \\
\hline \multicolumn{2}{|c|}{ Southern dry mixed deciduous forest } & \multicolumn{2}{|c|}{ Northern dry mixed deciduous forest } \\
\hline Degradation stages & \multicolumn{2}{|l|}{ Edaphic types } & Seral types \\
\hline Dry deciduous scrub & \multicolumn{2}{|c|}{ Anogeissus pendula forest } & Dry tropical riverain forest \\
\hline Dry savannah forest & \multicolumn{2}{|c|}{ Anogeissus pendula scrub } & $\begin{array}{l}\text { Khair (Acacia catechu)-sissoo (Dalbergia } \\
\text { sissoo) forest }\end{array}$ \\
\hline Dry grassland & \multicolumn{2}{|l|}{ Boswellia forest } & Inundation babul (Acacia nilotica) forest \\
\hline Euphorbia scrub & \multicolumn{2}{|c|}{$\begin{array}{l}\text { Babul (Acacia nilotica) forest Hardwickia forest Butea forest } \\
\text { Aegle forest, Laterite thorn forest, Saline/alkaline scrub } \\
\text { savannah, Phoenix savannah, Babul (Acacia nilotica) savannah, } \\
\text { Salvadora-Tamarix scrub, Dry bamboo brake }\end{array}$} & Secondary dry deciduous forest \\
\hline
\end{tabular}

Degradation stages, and edaphic and seral types occur in both southern and northern areas

experience extremely low temperature and humidity. These are stunted deciduous or evergreen forests with conifers. The alpine scrub vegetation comprises (i) moist-alpine scrub dominated by dwarf, evergreen shrubby conifers and broad-leaved species along with a prominent shrub layer; (ii) dry-alpine scrub comprising open formation of xerophytic scrubs with many herbs and grasses.

The Himalayan vegetation was divided into 11 forest formations by Singh and Singh (1987) on the basis of leaf characters (leaf drop pattern, leaf size, texture and shape), and elevation (Fig. 5 and Table 6). These authors have avoided using the term 'alpine', because they feel that the 'alpine' conditions in the Himalaya are not analogous to those of the Alps. The categories recognized by Champion and Seth (1968) that fall within the 11 formation types of Singh and Singh (1987) are also indicated in Table 6 and briefly described below.

Among the 11 forest formations, the submontane broadleaf ombrophilous forest has high species richness, multistratal structure, and abundance of buttressed trees $50 \mathrm{~m}$ or more tall. The common genera in this forest are Dipterocarpus, Artocarpus, Syzygium, Mesua, and Myristica. In the submontane seasonal broadleaf forest, about $60-90 \%$ of the top canopy is represented by Shorea robusta, which is the most extensively distributed and dominant species.

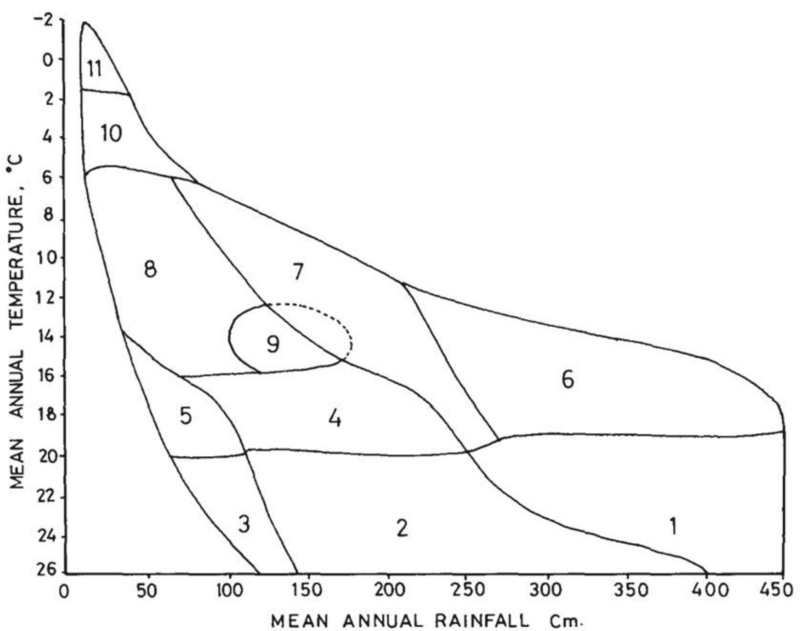

Fig. 5: Forest formations of the Himalaya in relation to rainfall and temperature. 1, Submontane broadleaf ombrophilous forest; 2, Submontane seasonal broadleaf forest; 3, Submontane broadleaf summer deciduous forest; 4, Low-montane needle-leaf forest with concentrated summer leaf-drop; 5, Low-montane sclerophyllous evergreen broadleaf forest; 6 , Midmontane broadleaf ombrophilous forest; 7, Low to mid-montane hemi-sclerophyllous broadleaf forest with concentrated summer leaf-drop; 8 , Mid-montane needle-leaf evergreen forest; 9, Mid-montane winter deciduous forest; 10, High-montane mixed stunted forest; and 11, Very high-montane scrub (Source: Singh and Singh, 1987)

The common canopy associates of sal in this forest are Schima wallichii, Stereospermum personatum, 
Table 6: Formation-types recognized in the Himalaya compared with the vegetation-types recognized by Champion and Seth (1968) (Source: Singh and Singh, 1987)

Formation-type

Submontane broadleaf ombrophilous forest

Submontane seasonal broadleaf forest

Submontane broadleaf summer-deciduous forest

Low-montane needle-leaf forest with concentrated summer leaf-drop

Low-montane sclerophyllous evergreen broadleaf forest

Mid-montane broadleaf ombrophilous forest

Low to mid-montane hemisclerophyllous broadleaf forest with concentrated summer leaf-drop

Mid-montane needle-leaf evergreen forest

Mid-montane winter-deciduous forest

High-montane mixed stunted forest

Very high-montane scrub
Equivalent groups, subgroups, and categories of Champion and Seth (1968)

Northern tropical wet evergreen forest (1B) and mesic part of northern tropical semi-evergreen forest (2B), lower part of northern subtropical broadleaved wet hill forest $(8 \mathrm{~B})$

Drier part of $2 \mathrm{~B}$ and moist parts of the mixed deciduous forest $\left(3 \mathrm{C} / \mathrm{C}_{3}\right)$

Northern dry mixed deciduous forest $\left(5 \mathrm{~B} / \mathrm{C}_{2}\right)$, and, dry Siwalik sal forest $\left(5 \mathrm{~B} / \mathrm{C}_{1 \mathrm{a}}\right)$, moist mixed deciduous forest $\left(3 \mathrm{C} / \mathrm{C}_{3}\right)$

Subtropical pine forest (9)

Subtropical dry evergreen forest $\left(10 \mathrm{C} / \mathrm{C}_{1}\right)$

East Himalayan wet temperate forest $\left(11 \mathrm{~B} / \mathrm{C}_{1}\right)$, higher part of northern subtropical broadleaf wet hill forest (8B)

Lower western Himalayan temperate forest $\left(12 / \mathrm{C}_{1}\right)$ and upper west Himalayan temperate forest $\left(12 / \mathrm{C}_{2}\right)$, excluding coniferous categories and deciduous category

Coniferous categories of lower western $\left(12 / \mathrm{C}_{1}\right)$ and upper west $\left(12 / \mathrm{C}_{2}\right)$ Himalayan temperate forests and east Himalayan moist temperate forest $\left(12 / \mathrm{C}_{3}\right)$

Moist temperate deciduous forest category of lower western Himalayan temperate forest $\left(12 / \mathrm{C}_{\mathrm{le}}\right)$

Subalpine forest (14)

Alpine scrub (15)
Sterculia spp., Caschela microcarpa, Lagerstroemia parviflora, Terminalia spp., Machilus villosa, and Anogeissus latifolia. In the submontane broadleaf summer deciduous forest, diversity is relatively high, and unlike the previous type, species dominance is not marked. In Bhabar of Kumaun Himalaya, Albizia procera, Adina cordifolia, Terminalia tomentosa, T. bellerica, Toona ciliata, and Anogeissus latifolia form the canopy, and Mallotus philippensis (a member of sal undercanopy) and Ougeinia oogeinensis form the understory tree layer. As recognized by Kenoyer (1921), a Bauhinia forest (common species, $B$. retusa, $B$. variegata, and $B$. vahli) with open canopy occur in the hills of Kumaun.

Low-montane needle-leaf forest with concentrated summer leaf drop formation type is represented by Pinus kesiya in Eastern Himalaya and $P$. roxburghii in Central and Western Himalaya. The shrub layer in this forest type is poor, and grasses predominate in the ground flora because of frequent burning (Saxena, 1979). Low-montane sclerophyllous evergreen broadleaf forest is dominated by Olea cuspidata and has abundant shrub layer. In the mid- montane broad leaf ombrophilous forest, none of the species generally attain more than $12 \%$ relative importance. Stratification is less developed and height of trees is lower. Common species are members of the Lauraceae, Machilus edulis, Michelia cathertii, Magnolia spp., Quercus lamellosa, Q. serrata, Castanopsis spp., Acer campbelli, etc. Low to midmontane hemi-sclerophyllous broadleaf forest with concentrated summer leaf drop type is species poor, rich in epiphytes, and generally has closed canopy (more than 80\%). Herb layer is less developed, without grasses, while the shrub growth is conspicuous. Due to predominance of generally one or two species, the dominant types are easily recognizable: (i) Quercus leucotrichophora (banj oak) forest, (ii) $Q$. lanuginosa (rianj oak) forest, (iii) $Q$. floribunda (tilonj oak) forest, (iv) $Q$. semecarpifolia (kharsu oak) forest, and (v) $Q$. leucotrichophora-Q, floribunda forest. These dominant types exhibit a remarkable feature of altitudinal variation in oak species.

Mid-montane needle-leaf evergreen forest is mostly dominated by needle-leaved species, viz., Cedrus deodara (deodar) and Pinus wallichiana 
(blue pine). Western part mostly contains Abies pindrow (silver fir) and Picea smithiana (spruce), and the eastern part has Abies delavayi, A. hylium, and Tsuga dumosa (hemlock) among the dominants. Mid-montane winter deciduous forest occurs in the region occupied by mid-montane hemi-sclerophyllous and needle-leaf evergreen forests. This forest type generally occupies the moist places of limited areas along the streams. The common species are Aesculus indica, Acer pictum, A. caesium, Carpinus viminea, Ulmus wallichiana, Betula alnoides, Pyrus lanata, Juglans regia, and Fraxinus micrantha. In the highmontane mixed stunted forest (above $3000 \mathrm{~m}$ ), plants mainly depend on snowmelt for their water requirement, as the effect of monsoon is minimum. In the central and western Himalaya, the common species are deciduous birch or bhojpatra (Betula utilis), evergreen fir (Abies spectabilis), evergreen oak ( $Q$. semecarpifolia). In the lower canopy, Rhododendron campanulatum (3-10 m) is the most common species. In the last formation type, i.e., very high-montane scrub (above $3500 \mathrm{~m}$ and up to 4900 $\mathrm{m})$, all taxa of the above mentioned formations are present except for the Abies species. The common herbaceous associates in this type are species of Caragana and Artemisia.

\section{Grassland Ecosystems}

The grassland ecosystems in many parts of India have originated due to destruction of natural forests by biotic interference, particularly due to excessive grazing and land clearing for agriculture (http://environmentof earth.wordpress.com/tag/vegetation). Dabadghao and Shankarnarayan (1973) identified five major grass cover types (Table 7). According to Whyte (1968), the distribution of these grass cover types is primarily governed by climatic factors, and chiefly by latitude. Thus, while Sehima-Dichanthium cover is tropical, the Dichanthium-Cenchrus-Lasiurus, PhragmitesSaccharum-Imperata and Themeda-Arundinella covers are distinctly sub-tropical. The temperatealpine cover is distinct from the above types. The altitude separates the Themeda-Arundinella grass cover which is restricted to the northern hills from Phragmites-Saccharum-Imperata and from Dichanthium-Cenchrus-Lasiurus types which occur in plains. In the plains, moisture conditions separate Phragmites-Saccharum-Imperata type (moist to wet habitats) from the Dichanthium-Cenchrus-Lasiurus type (relatively dry habitats).

Whyte (1968) has also maintained a Nilgiri highaltitude grass cover type dominated by Andropogon polyptychus and Chrysopogon zeylanicus which represents a sub-climax stabilized by fire, grazing and erosion.

The areas occupied by the five grass cover types as shown by Dabadghao and Shankarnarayan (1973) represent the potential area; however, much of the area shown under each grass cover type is presently either under forest or under cultivation (Yadava and Singh, 1977). Further, due to local variations in climate, topography, soil and biotic pressure, a myriad of distinct or transitional communities occur within the potential area of each of the above major grassland types (Yadava and Singh, 1977; Singh and Gupta, 1993).

\section{Wetland Ecosystems}

The inland aquatic ecosystems are also very diverse and include rivers, streams, springs, lakes, lagoons, estuaries and various human made habitats as rivers, canals, tanks/ponds (Gopal, 2013). The country is covered by a network of 14 major, 44 medium and several minor rivers and their tributaries (Rao, 1975). Natural lakes are few and cover about 0.72 million ha (Jhingran, 1991), but > 4290 large and numerous smaller reservoirs cover more than 3.15 million hectares (Sugunan, 1995; Suryanarayanan, 1996). These water bodies are supplemented by thousands of temple tanks, oxbow lakes and village ponds. Jhingran (1991) estimates 1.81 million ha of oxbow lakes and 2.75 million ha peninsular tanks. Other floodplain and seasonal shallow water habitats may account for another 1.5 million ha. The coastal mangroves ( 0.4 million ha), estuaries ( 3.9 million ha) and lagoons and backwaters (3.5 million ha) lying inland (behind coastline) account for further 8 million ha; thus, the total area of inland aquatic ecosystems (excluding cultivated paddy fields) is estimated at about 18 million ha (Gopal, 1997).

Depending upon the duration of water logging, Gopal (1987) has grouped Indian fresh water wetlands into two major types: (i) the perennial wetlands, with water logging throughout the year, include habitats such as river banks and margins of large lakes or reservoirs, and (ii) seasonal wetlands which dry up 
Table 7: Major grassland types of India (based on Dabadghao and Shankarnarayan, 1973)

\begin{tabular}{|c|c|c|}
\hline Grassland types & Characteristics & Occurrence \\
\hline Sehima-Dichanthium & $\begin{array}{l}\text { topography undulating, rainfall } 300-6350 \mathrm{~mm} \text {; develop on sandy-loam soils; } \\
\text { dominant component comprises } 24 \text { perennial grasses along with } 129 \text { herbaceous } \\
\text { species including } 56 \text { legumes; on gravelly soils and under protection or light grazing } \\
\text { Sehima dominates and on level soils Dichanthium is the dominant grass }\end{array}$ & $\begin{array}{l}\text { peninsular India including } \\
\text { the central Indian plateau, } \\
\text { Chhota Nagpur plateau } \\
\text { and the Aravalli ranges }\end{array}$ \\
\hline $\begin{array}{l}\text { Dichanthium- } \\
\text { Cenchrus-Lasiurus }\end{array}$ & $\begin{array}{l}\text { topography broken here and there by spurs of southern hill ranges and by sand } \\
\text { dunes in western Rajasthan, monsoon rainfall } 100-700 \mathrm{~mm} \text {; eleven important } \\
\text { perennial grasses with } 45 \text { herbaceous species including } 19 \text { legumes; Cenchurus } \\
\text { setigerus and C. cilliaris from communities where soil is not too dry, and Lasiurus } \\
\text { indicus on loose sandy soil in areas receiving less than } 300 \mathrm{~mm} \text { rainfall }\end{array}$ & $\begin{array}{l}\text { northern portion of } \\
\text { Gujarat, whole of } \\
\text { Rajasthan, western } \\
\text { Uttar Pradesh and Punjab }\end{array}$ \\
\hline $\begin{array}{l}\text { Phragmites- } \\
\text { Saccharum-Imperata }\end{array}$ & $\begin{array}{l}\text { topography level characterized by low-lying, ill-drained soils; } 19 \text { principal grass } \\
\text { species and } 56 \text { herbs including } 16 \text { legumes predominate }\end{array}$ & $\begin{array}{l}\text { part of Gangetic plains, } \\
\text { the Brahmaputra valley and } \\
\text { extends to the plains of } \\
\text { Punjab }\end{array}$ \\
\hline Themida-Arundinella & $\begin{array}{l}\text { rainfall } 1000-2000 \mathrm{~mm} \text {, snow-fall in winter towards upper ranges; } 16 \text { perennial } \\
\text { grasses (including species of Bothriochloa, Chrysopogon and Cymbopogon) and } \\
34 \text { herbaceous species including } 9 \text { legumes, are important constituents }\end{array}$ & $\begin{array}{l}\text { appears around the altitude } \\
350 \mathrm{~m} \text { and extends upto } \\
2100 \mathrm{~m}\end{array}$ \\
\hline Temperate-Alpine & $\begin{array}{l}35 \text { important perennial grasses (including species of Agrostis, Danthonia, } \\
\text { Phleum and Poa), } 68 \text { herbaceous species including } 6 \text { legumes are important } \\
\text { vegetation components }\end{array}$ & $\begin{array}{l}\text { occurs above } 2100 \mathrm{~m} \text { in } \\
\text { northern montane belt and } \\
\text { above } 1500 \mathrm{~m} \text { in the eastern } \\
\text { belt, snow-fall is common }\end{array}$ \\
\hline
\end{tabular}

completely for varying periods of time depending upon the vagaries of monsoon, examples are numerous village ponds, fish ponds, paddy fields, river flood plains, etc. Biswas (1984) has reported 1193 wetlands in India covering a total area of 3.9 million hectares in 274 districts of India.

The aquatic ecosystems including wetlands support diverse communities which harbour about 1800 algal species and 1250 higher plant species (Gopal, 2013). The aquatic (and wetland) flora (including algae) constitute more than $15 \%$ of the India's total estimated floristic diversity (Gopal, 1997). The aquatic faunal component is also prominent. According to Gopal (2013), there are about 300 Rotifera, 285 Mollusca, 100 Cladocera, 100 Ostracoda, > 300 Copepoda, 742 fishes, and about 1000 Aves. The species diversity of fishes, birds and other fauna is very high in many aquatic habitats. For example, there are more than 330 species of birds but fewer species of fishes in Keoladev National Park (Vijayan, 1995), 64 fish species and 116 species of birds in Loktak lake (Singh and Singh, 1994), and 217 fish and 160 bird species in Lake Chilika (Ram et al., 1994).

\section{Mangrove Ecosystems}

The term mangrove refers to an ecological group of halophytic plant species as well as to a variety of complex plant communities dominated by these species (Upadhyay et al., 2002). The coastal zone of the mainland of India and that of Andaman and Nicobar Islands is endowed with the presence of extensive and diverse mangroves (MoEF, 2009). About $5 \%$ of the world's mangrove vegetation at the global level has been reported from India which accounts for $0.67 \%$ of the total designated forest area of the country and is spread over an area of $4,662.56 \mathrm{~km}^{2}$ along the coastal States/UTs (Das Gupta and Shaw, 2013). Over the past two decades (1987 to 2011), the mangrove forest cover has increased by $616.56 \mathrm{~km}^{2}$ (FSI, 2016). The very dense mangrove comprises $1403 \mathrm{~km}^{2}$ (30.10\% of the mangrove cover), moderately dense mangrove is $1658.12 \mathrm{~km}^{2}(35.57 \%$ of the mangrove cover) while open mangroves cover an area of $1600.44 \mathrm{~km}^{2}(34.33 \%$ of the mangrove cover) (MoEF, 2012). West Bengal has the maximum of mangrove cover in the country, followed by Gujarat and Andaman and Nicobar Islands.

Mangrove ecosystems are among the most productive and biologically diverse ecosystems and 
constitute a bridge between terrestrial and marine ecosystems (MoEF, 2012). The dominant salt-tolerant, sclerophyllous broadleaved trees form a unique ecosystem with associated plants, including epiphytic and terrestrial ferns, orchids, lichens, non-mangrove halophytes, sea grasses and seaweeds, and fauna such as fish, shrimp, shellfish, crabs, lobsters, reptiles and birds (Upadhyay et al., 2002). These ecosystems are found in the inter-tidal zones of sheltered shores, estuaries, creeks, backwaters, lagoons, marshes and mud-flats. Mangroves provide habitats, spawning grounds, nurseries and nutrients to a number of animals (MoEF, 2009) and harbour several endangered species ranging from reptiles (e.g. crocodiles, iguanas and snakes) and amphibians, to mammals (tigers, deer, otters and dolphins) and birds (herons, egrets, pelicans and eagles). One hundred and sixteen plant species have been recorded which include 59 mangrove species, 47 algae and 10 species of sea grasses (Banerjee et al., 1989). About 65 vascular plant species belonging to 31 families and 59 genera have been reported from the mangrove ecosystems of India (Banerjee and Ghosh, 1998). Faunal diversity in mangrove forests of India is also immense, for example, number of species of Crustaceans 229, Molluscs 212, Wood borers 25, Fishes 185, Reptiles 39, Birds 117, Mammals 36 , Microbenthos 111, Shellfishes 20 (Jagtap, 1994).

The number and dominant species of major mangrove wetlands of India are shown in Table 8.

\section{Marine and Coastal Ecosystems}

As elsewhere in the world, the extent of marine biodiversity in India is relatively less known (MoEF, 2009). More than 13,000 coastal and marine species have been reported from India. The total area of coral reefs comprises 2,375 sq km (Ministry of Earth Sciences and Space Application Centre, 1997). Major coral reef ecosystems are in Gulf of Mannar, Gulf of Kachchh, Andaman and Nicobar, and Lakshadweep Islands and embrace all the three major reef types (atoll, fringing and barrier) (MoEF, 2009).

According to India's fourth National Report to the Convention on Biological Diversity (CBD) (MoEF, 2009), the Andaman Islands have around $80 \%$ of the global coral diversity, suggesting that a final count could reach up to 400 species. These include 15 families, 60 genera and 208 species of Scleractinia (reef building and hermatypic corals) from four major reefs of India viz., Gulf of Kachchh (36 species, 20 genera) Lakshadweep (91 species, 34 genera), Gulf of Mannar and Palk Bay (82 species 27 genera) Andaman and Nicobar Islands (177 species, 57 genera).

\section{Agro-Ecosystems}

India has a variety of distinct agro-ecosystems, characterized by variations in edaphic, climatic and geographic features, and consequently a diverse cropping pattern and crop composition. The country is one of the eight Vavilov's centers of origin of

Table 8: Characteristics of major mangrove wetlands of India (Source: Selvam, 2003)

\begin{tabular}{lccl}
\hline Mangrove & $\begin{array}{c}\text { Total rainfall } \\
(\mathrm{mm})\end{array}$ & $\begin{array}{c}\text { No. of } \\
\text { species }\end{array}$ & Dominant species \\
\hline East coast of India & 1600 to 1800 & 26 & Excoecaria agallacha, Ceriops decandra and Sonneratia apetala \\
Sunderbans & 1600 & 26 & Excoecaria agallacha, Ceriops decandra and Sonneratia apetala \\
Mahanadi & 1100 & 17 & Excoecaria agallacha, Avicennia officinalis and Sonneratia apetala \\
Godavari & 1200 & 12 & Avicennia marina \\
Krishna & 1300 & 11 & Avicennia marina \\
Pichavaram and Muthupet & 470 & 8 & Avicennia marina \\
West coast of India & 900 & 8 & Avicennia marina \\
Gulf of India & 2750 to 3080 & 24 & Rhizophora apiculata, Rhizophora mucronata and Ceriops tagal \\
Gulf of Khambat & &
\end{tabular}


cultivated plants in the world. India has more than 800 crop species, each with immense variation, for example rice has 50,000 varieties and sorghum 5,000 varieties. Needless to say, a wide variety of weeds make a part of the agro-ecosystems. The National Bureau of Soil Survey and Land Use Planning distinguished 20 broad agro ecological zones, based on natural features and growing periods (Table 9). This large variety of ecological zones signifies the distinct agro-ecosystems.

No discussion on agro-ecosystems can ignore the shifting or slash and burn agriculture, so predominant in the northern India. Shifting cultivation is one of the oldest farming systems, believed to have originated in the Neolithic period around 7000 B.C., and is still practiced in the states of Andhra Pradesh, Nagaland, Meghalaya, Mizoram, Manipur, Tripura, Arunachal Pradesh, Kerala, Karnataka and Orissa, by about 5 million tribal families on $4.37 \mathrm{mha}$. Shifting cultivation consists of clearing a plot of forest land by burning the vegetation and cultivating for one or two years. Subsequently, the old cultivated plot is left fallow for a variable period of time, to allow for the recovery of the vegetation, and then again it is cleared by burning and cultivated. The practice has been extensively documented (see Ramakrishnan, 1992; Ramakrishnan, 2015; Ranjan and Upadhyay, 1999; Shillong Declaration, 2004; Tiwari, 2007; Deb et al., 2013). Because of the increasing population and increasing demand for land for cultivation, the fallow period has declined from 25-30 years to 2-3 years. This has resulted in large-scale deforestation, soil and nutrient loss, and invasion by weeds and other species (see Ranjan and Upadhyay, 1999, Deb et al., 2013, Ramakrishnan, 2015). The shifting cultivation practices vary from tribe to tribe in terms of land tenure, fallow period, species cultivated and innovations. Table10 gives an example of three tribes in Meghalaya.

\section{Human Impacts}

The growing human population has exerted a significant impact on all ecosystem types in all regions of India. High human and livestock demand for food, fodder and firewood has resulted in overexploitation of terrestrial as well as aquatic ecosystems. Wind and water erosion, water logging and problems of salinity and alkalinity have resulted into land degradation of at least one third of the geographical area. Human activities have also resulted into high air and water pollution. India now has 321 globally threatened floral and 614 faunal species as per the IUCN (2012) Red List.

There has been a conspicuous change in landuse pattern, habitat loss and forest fragmentation. For example, Jha et al. (2005) observed in the Vindhyan dry tropical forest an increase in number of patches formed due to forest fragmentation with corresponding decrease in mean patch area and mean patch perimeter during a ten year period. The large forest fragments contained the greatest number of tree species. Sagar and Singh (2004) have reported that about $52 \%$ of the total 65 species inventoried in a dry tropical forest, exhibited local demographic instability. The increase in the proportion of declining species with increase in disturbance intensity indicated that local anthropogenic pressure was responsible for the depression. Sagar and Singh (2005) observed a conspicuous decline in species richness and alpha diversity due to biotic disturbance in the forest (Fig. 6) Chaturvedi et al. (2017b). Large tracks of natural forests have been converted into cultivated land or monoculture of cash and timber crops such as tea, coffee, rubber, teak and eucalyptus.

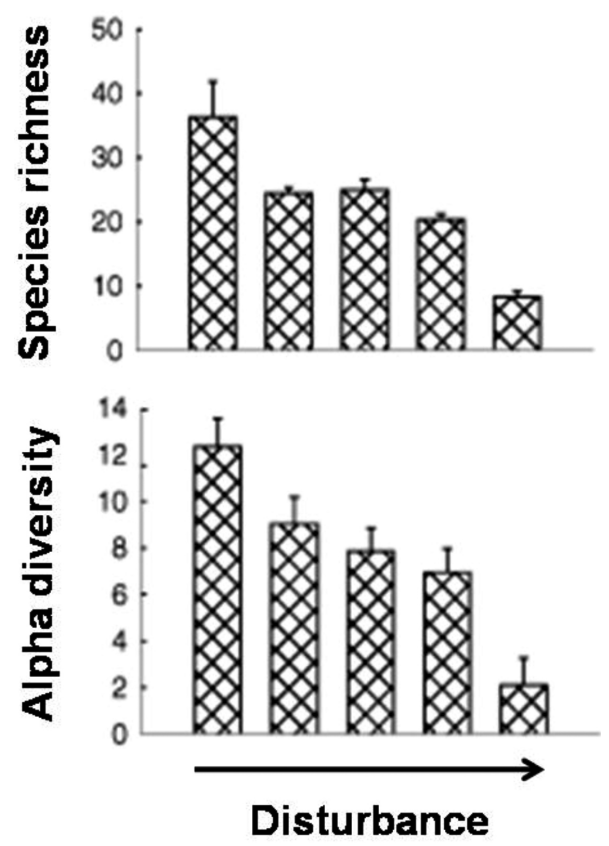

Fig. 6: Species richness and alpha diversity in a gradient of disturbance (from least to highest) in the dry tropical forest region (Source: Sagar and Singh, 2005) 
Table 9: Agro-ecological regions in India

Agro-ecological regions
Arid Ecosystem
Western Himalayas (Eastern and Western aspect of Ladakh Plateau,
north Kashmir Himalayas)

Western Plain, Kachchh and part of Kathiawar Peninsula (Rajasthan Bagar, North Gujarat Plain and South-Western Punjab Plain, Kachchh and North Kathiawar Peninsula)

Deccan plateau (Karnataka Plateau)

\section{Semi-arid Ecosystem}

Northern Plain (and Central Highlands) including Aravallis (North Punjab Plain, Ganga-Yamuna Doab and Rajasthan Upland, North Gujarat Plain, Rohilkhand and Avadah Plain, Madhya Bharat Plateau and Bundelkhand Uplands)

Central (Malwa) Highlands, Gujarat plains and Kathiawar Peninsula (Coastal and Central Kathiawar Peninsula, Madhya Bharat Plateau, Western Malwa Plateau, Eastern Gujarat Plain, Vindhyan and Satpura range and Narmada Valley)

Deccan Plateau (Central, Eastern and Western Maharashtra Plateau, North and Western Karnataka Plateau, North Western Telangana Plateau, North Sahyadris)

Deccan Plateau (Telangana) and Eastern Ghats (North and South Telangana Plateau, Eastern Ghat)

Eastern Ghats and Tamil Nadu Uplands and Deccan (Karnataka) Plateau hot dry/moist semi-arid; red loamy (Tamil Nadu Uplands and Plains, Leeward Flanks of South Sahyadris, soils; GP 90-150 days Central Karnataka Plateau)

\section{Sub-humid Ecosystem}

Northern Plain (Punjab, Rohilkhand, Avadh and south Bihar Plains)

Central Highlands (Malwa and Bundelkhand) (Malwa Plateau, Vindhyan Scarpland and Narmada Valley, Satpura range and Eastern Maharashtra Plateau, Bundelkhand Plateau, Wainganga Valley)

Chhattisgarh/Mahanadi Basin (Chhattisgarh/Mahanadi Basin)

Eastern Plateau (Chhotanagpur) and Eastern Ghats (Garjat Hills, Dandakaranya and Eastern Ghats, Chhotanagpur Plateau)

Eastern Plain (North Bihar and Avadh Plains, Foothills of Central Himalayas)

Western Himalayas (South Kashmir, Himachal Pradesh, Punjab and Kumaun Himalayas)

\section{Humid-perhumid Ecosystem}

Assam and Bengal Plain (Middle, Lower and Upper Brahmaputra Plain, Bengal basin, North Bihar Plain, Teesta, Barak Valley)

Descriptive features

cold, hyper-arid; shallow skeletal soils; length of Growing Period

(GP) $<90$ days

hot, hyper-arid; desert and saline soils; GP $<90$ days

hot arid; red and black soils; GP $<90$ days

hot dry/moist semi-arid; alluvium derived soils; GP 90-150 days

hot dry/moist semi-arid; medium and deep black soils;

GP 90-150 days

hot dry/moist semi-arid, hot subhumid; shallow and medium (with inclusion of deep) black soils; GP 90-150 days

hot dry/moist semi-arid/dry subhumid; red and black soils; GP 90-150 days derived soils; GP 150-180 days and red soils; GP 150-180 (to 210) days yellow soils; GP 150-180 days

hot dry/moist sub-humid; red and lateritic soils; GP 150-180 (to 210) days

hot/warm dry/moist sub-humid; alluvium-derived soils; GP 180-210 days brown forest and podzolic soils;

GP 180-210 + days

Major crops

millets, barley and wheat

millets, cotton and oil seeds

millets and pulses

millets, wheat, pulses, maize, sugarcane and cotton

millets, wheat and pulses

millets, cotton, pulses and sugarcane

millets, oilseeds, rice, cotton and sugarcane

oilseeds, rice, cotton and sugarcane

hot dry/moist sub-humid; alluvium- pulses and sugarcane

hot dry/moist sub-humid; black sorghum and pulses

hot dry/moist sub-humid; red and rice, millets and wheat rice, pulses and millets

rice, wheat and sugarcane

cold and warm by dry semi-arid/dry wheat, millets, maize sub-humid, warm moist sub-humid; and rice

hot/warm moist sub-humid/ humid; alluvium-derived soils; GP 210 + days rice, jute and plantation crops 
Eastern Himalayas (Bhutan foot-hills, Darjeeling and Sikkim Himalayas, warm to hot per-humid; brown Arunachal Pradesh, Manipur, Mizoram, Tripura)

North-eastern Hills (Purvachal) (Meghalaya Plateau, Nagaland Hill, Purvachal)

\section{Coastal Ecosystem}

Eastern Coastal Plain (South and North Tamil Nadu Plains (Coastal), Andhra Plain, Utkal Plain, East Godavari Delta, Gangetic Delta)

Western Ghats and Coastal Plain (Central, North and South Sahyadris and Konkan Coast, Karnataka and Kerala Coastal plain) and red hill soils; GP $210+$ days

warm to hot moist humid to per- forest and rice in patches humid; red and lateritic soils;

GP $210+$ days rice and millets

hot dry/moist semi-arid/sub-humid/ rice, pulses and millets humid; coastal alluvium-derived

soils; GP 90-210 + days

hot moist sub-humid to humid; red, lateritic and alluvium- derived soils; GP 219 + days

hot per-humid/humid; red loamy and sandy soils; GP $210+$ days rice, tapioca, coconut and millets

forest, coconut and rice

\section{Island Ecosystem}

Islands of Andaman-Nicobar and Lakshadweep (Andaman and Nicobar group of Islands, Level Lakshadweep and group of Island)

On account of anthropogenic factors

River systems have been greatly affected by building dams and reservoirs. A study in the Himalayan region (Pandit and Grumbine, 2012) has reported that the ongoing and proposed hydropower development would adversely affect the persistence of species across the taxonomic groups. The study emphasized that the proposed dam building would affect nearly $90 \%$ of Indian Himalayan valleys, and by 2025 , about 22 angiosperm and 7 vertebrate taxa might become extinct. Due to disturbance in the dense forests caused by dam building, a reduction of $35 \%$ tree species richness, $42 \%$ tree density, and $30 \%$ tree basal cover is expected in the Himalayan region (Pandit and Grumbine, 2012).

The wetland ecosystems are threatened by discharge of waste effluents, surface runoff, weed infestation and uncontrolled siltation. Overfishing has resulted into a decline of fishery resources of fresh and marine water bodies. Changes in temperature, precipitation, evapo-transpiration and run-off have been suggested to alter the hydrological regime of the inland natural wetlands, particularly in arid and semi-arid regions (Patel et al., 2009). Few studies have reported that some of the most sensitive high altitude and coastal wetlands of India including mangroves and coral reefs are likely to be affected by climate change (Patel et al., 2009). It has been estimated that the climate change induced sea level rise of $1 \mathrm{~m}$ could result in the loss of $84 \%$ of coastal wetlands and $13 \%$ of saline wetlands of India (Blankespoor et al., 2012; Bassi et al., 2014). (reclamation of land, discharge of wastes, etc.) and natural factors like global warming, mangrove ecosystems are presently one of the most threatened ecosystems (MoEF, 2012). Due to diversion of freshwater in the upstream area, the periodicity and quantity of freshwater reaching the mangrove environment has been reduced leading to a dramatic change in the floral diversity of the mangrove wetlands (Selvam, 2003). Some of the mangrove species are on the way to extinction from the west coast, examples are Xylocarpus granatum, Bruguiera cylindrica, Sonneratia acida and Cynometra ramiflora. Similarly, Nypa fruticans, Heritiera minor and $H$. formes have all but disappeared from the Sunderbans (Upadhyay et al., 2002). The current status of all mangrove wetlands in India, except those of Andaman and Nicobar islands, indicates that the species having low-saline tolerance are gradually disappearing and the species like Avicennia marina exhibiting broad range of salinity tolerance are becoming dominant (Selvam, 2003).

The disturbance of terrestrial ecosystems has seen the spread of invasive alien species degrading the health of natural as well as manmade ecosystems. Biotic invasions interfere with the socio-economic system, food security, human and animal health and cause enormous harm of the region (Kohli et al., 2012). Other environmental problems such as landscape change, disturbance and climate change further increase the biotic invasion process (Kohli et $a l ., 2012)$. In the north-eastern hill region of India, 
Table 10: Salient features of Shifting cultivation practiced by Khasi, Karbi and Garo communities of Meghalaya (Source: Deb et al., 2013)

\begin{tabular}{|c|c|c|c|}
\hline \multirow[t]{2}{*}{ Activities } & \multirow[b]{2}{*}{ Khasi } & \multicolumn{2}{|l|}{ Name of the tribes } \\
\hline & & Karbi & Garo \\
\hline Land ownership & Private/Rented & Private & Community \\
\hline $\begin{array}{l}\text { Allotment of plot by } \\
\text { traditional institution }\end{array}$ & Not necessary & Not necessary & Practiced \\
\hline $\begin{array}{l}\text { Labour for slashing } \\
\text { and burning }\end{array}$ & Family members & Family members & Community activity \\
\hline Burning Months & February-March & April & March \\
\hline Sowing Months & March-May & April-June & March-April \\
\hline No. of crops grown & $14-16$ & 24 & $30-35$ \\
\hline $\begin{array}{l}\text { Dominant subsistence } \\
\text { crops }\end{array}$ & $\begin{array}{l}\text { Bean, Bitter gourd, Coix } \\
\text { (Sohriew), Cucumber, Soyabean, } \\
\text { Dioscorea, Pea, Raddish, Potato, } \\
\text { Sweet Tapioca and etc. }\end{array}$ & $\begin{array}{l}\text { Bean, Bitter gourd, Cucumber, } \\
\text { Jhika (fruit), Lady's finger, Lao, } \\
\text { Maize, Mesta, Sponge gourd, } \\
\text { Banana, Paddy, Sweet Potato, } \\
\text { Tapioca and Turmeric }\end{array}$ & $\begin{array}{l}\text { Brinjal, Lai sak (leaf), Local lettuce, } \\
\text { Millet, mustard, Sesame, Bean, } \\
\text { Bilik (legume), Cotton, Cucumber, } \\
\text { Gourd, Kharek (fruit), Jingka } \\
\text { (fruit), Lady's finger, Lau, Lentil, } \\
\text { Melon, Mesta, Paddy, Cauliflower } \\
\text { Pumpkin }\end{array}$ \\
\hline Cash crops & Turmeric, Potato, Maize, Colocassia & $\begin{array}{l}\text { Potato, Colocassia, Pumpkin, } \\
\text { Chilly, Ginger }\end{array}$ & Ginger, Maize, Chilly, Pumpkin \\
\hline Fruit trees & $\begin{array}{l}\text { Artocarpus chaplasha, Citrus sp., } \\
\text { Litchi chinensis, Mangifera indica, } \\
\text { Myrica esculenta, Prunus nepalensis, } \\
\text { Musa sp. }\end{array}$ & Artocarpus sp. & Musa sp., Citrus sp. \\
\hline Fuel wood trees & $\begin{array}{l}\text { Artocarpus sp., Betula alnoides, } \\
\text { Castanopsis tribuloides, Ficus } \\
\text { bengalensis, Litchi chinensis, } \\
\text { Mallotus nepalensis, Mangifera- } \\
\text { indica, Myrica esculenta, Prunus } \\
\text { nepalensis, Quercus sp. and Schima } \\
\text { wallichi }\end{array}$ & $\begin{array}{l}\text { Albizia procera, Artocarpus sp., } \\
\text { Cassia fistula, Duabanga grandi- } \\
\text { flora, Gmelina arborea, Michelia } \\
\text { champaca, Shorea robusta, } \\
\text { Tectona grandis and Toona } \\
\text { ciliata }\end{array}$ & $\begin{array}{l}\text { Actinodaphne obovata, Albizia } \\
\text { chinensis, Albizia odoratissima, } \\
\text { Callicarpa arborea, Careya } \\
\text { arborea, Duabanga grandiflora, } \\
\text { Eurya japonica, Gmelina } \\
\text { arborea, Macaranga sp. and } \\
\text { Trema orientalis }\end{array}$ \\
\hline Weeding & Twice & Once & Twice \\
\hline Harvesting period & June-July, Nov-Jan & June-Feb & June-Feb \\
\hline Cropping period (years) & 1 & 1 & $1-3$ \\
\hline Fallow period (years) & $8-15$ & $3-5$ & $7-9$ \\
\hline Production $\left(\mathrm{kg} \mathrm{ha}^{-1}\right)$ & 4653 & 1991 & 11293 \\
\hline
\end{tabular}

Kushwaha et al. (1981) observed that due to increased population pressure and reduction in the availability of cultivation land, people have reduced the fallow period in the shifting agriculture to about 45 years, which has led to enormous increase in the population of noxious weeds like Chromolaena odorata. In India, about $18 \%$ flora is composed of adventive aliens, which have caused havoc in the terrestrial and aquatic ecosystems (Raghubanshi et al., 2005). Important invasive plants in India are listed in Table 11.

\section{Ecosystem Conservation Scenario}

India has taken significant steps for conservation of its biodiversity. We have a network of 683 Proctected areas for in-situ conservation (National Parks 102, Wildlife Sanctuaries 520, Conservation Reserves 57 and Community Reserves 4, established under the Wildlife (Protection) Act, 1972) covering a total area of $164980.75 \mathrm{~km}^{2}$ (5.02\% of the total geographical area of the country) (http://wiienvis.nic.in/Database/ Protected_Area_854.aspx). India also has special 
Table 11: List of Prominent Invasive Alien Plants in Indian Subcontinent (Arranged as per alphabetic order of family) (Source: Kohli et al., 2012)

\begin{tabular}{|c|c|c|c|c|}
\hline S.No. & Family name & Botanical and (Common name in parentheses) & Nativity & Life-form \\
\hline 1. & Amaranthaceae & $\begin{array}{l}\text { Chenopodium album (Lamb's-quarters) } \\
\text { Alternanthera philoxeroides (Alligator weed) }\end{array}$ & $\begin{array}{l}\text { Europe } \\
\text { South America }\end{array}$ & $\begin{array}{l}\text { Herb } \\
\text { Aquatic herb }\end{array}$ \\
\hline 2. & Amaryllidaceae & Zephyranthes citrina (Yellow rain lily) & Cent. South America & Herb \\
\hline 3. & Apocynaceae & Cryptostegia grandiflora (Rubber vine) & Madagascar & Vine-climber \\
\hline 4. & Araceae & Pistia stratiotes (Tropical duckweed) & South America & Aquatic Plant \\
\hline 5. & Asparagaceae & Asparagus densiflorus (Asparagus fern) & South Africa & Herb \\
\hline 6. & Asteraceae & $\begin{array}{l}\text { Ageratum conyzoides (Billy goat weed) } \\
\text { Ageratum houstonianum (Flossflower, Bluemink) } \\
\text { Ambrosia artemisiifolia (Small ragweed) } \\
\text { Anthemis cotula (Stinking mayweed) } \\
\text { Chromolaena odoratum (Siam weed) } \\
\text { Cirsium arvense (Creeping thistle) } \\
\text { Eupatorium adenophorum (Crofton weed) } \\
\text { Eupatorium cannabinum (Hemp-Agrimony) } \\
\text { Gymnocoronis spilanthoides (Senegal tea plant) } \\
\text { Leucanthemum vulgare (Oxe-eye daisy) } \\
\text { Mikania micrantha (Mile-a-minute weed) } \\
\text { Parthenium hysterophorus (Ragweed parthenium) } \\
\text { Sphagneticola trilobata (Singapore daisy) } \\
\text { Synedrella vialis (Straggler daisy) } \\
\text { Tagetes minuta (Mexican marigold) }\end{array}$ & $\begin{array}{l}\text { Tropical America } \\
\text { Central America, Mexico } \\
\text { USA, Canada, Mexico } \\
\text { Europe } \\
\text { Cent. South America } \\
\text { Europe } \\
\text { Central America } \\
\text { British Isles } \\
\text { South America } \\
\text { Europe } \\
\text { Cent. South America } \\
\text { Tropical America } \\
\text { Central America } \\
\text { South America } \\
\text { South America }\end{array}$ & $\begin{array}{l}\text { Herb } \\
\text { Herb } \\
\text { Herb } \\
\text { Herb } \\
\text { Shrub } \\
\text { Herb } \\
\text { Shrub } \\
\text { Herb } \\
\text { Aquaticplant } \\
\text { Herb } \\
\text { Vine/Climber } \\
\text { Herb } \\
\text { Herb } \\
\text { Herb } \\
\text { Herb }\end{array}$ \\
\hline 7. & Azollaceae & Azolla pinnata (Mosquito fern) & Not Specific & Aquatic plant \\
\hline 8. & Bignoniaceae & Macfadyena unguis-cati (Cat's claw vine) & Central America & Climber \\
\hline 9. & Cabombaceae & Cabomba caroliniana (Green cabomba) & South America & Aquatic plant \\
\hline 10. & Convolvulaceae & $\begin{array}{l}\text { Ipomoea aquatica (Water spinach) } \\
\text { Merremia peltata (Merremia) }\end{array}$ & $\begin{array}{l}\text { China } \\
\text { Africa }\end{array}$ & $\begin{array}{l}\text { Climber } \\
\text { Climber }\end{array}$ \\
\hline 11. & Elaeagnaceae & Elaeagnus umbellate (Japanese Silverberry) & China, Korea, Japan & Tree/Shrub \\
\hline 12. & Euphorbiaceae & $\begin{array}{l}\text { Ricinus communis (Castor bean) } \\
\text { Sapium sebiferum (Chinese tallow) }\end{array}$ & $\begin{array}{l}\text { Northeastern Africa } \\
\text { China }\end{array}$ & $\begin{array}{l}\text { Tree/Shrub } \\
\text { Tree }\end{array}$ \\
\hline 13. & Fabaceae & $\begin{array}{l}\text { Acacia farnesiana (Sweet acacia) } \\
\text { Acacia mearnsii (Black wattle) } \\
\text { Acacia melanoxylon (Blackwood acacia) } \\
\text { Leucaena leucocephala (Wild tamarind) } \\
\text { Mimosa diplotricha (Giant sensitive plant) } \\
\text { Mimosa pudica (Touch-me-not) } \\
\text { Prosopis juliflora (Mesquite) } \\
\text { Ulex europeus (Gorse) }\end{array}$ & $\begin{array}{l}\text { Tropical America } \\
\text { Australia } \\
\text { Australia } \\
\text { Tropical America } \\
\text { South America } \\
\text { South America } \\
\text { Cent. South America } \\
\text { Europe }\end{array}$ & $\begin{array}{l}\text { Tree/Shrub } \\
\text { Tree } \\
\text { Tree } \\
\text { Tree } \\
\text { Climber/Shrub } \\
\text { Herb } \\
\text { Tree } \\
\text { Tree }\end{array}$ \\
\hline 14. & Hydrocharitaceae & Hydrilla verticillata (Water thyme) & Asia, North Australia & Aquatic plant \\
\hline 15. & Limnocharitaceae & Limnocharis flava (Yellow velvetleaf) & South America & Aquatic plant \\
\hline 16. & Melastomataceae & $\begin{array}{l}\text { Clidemia hirta (Koster's curse) Miconia calvescens } \\
\text { (Velvet tree) }\end{array}$ & $\begin{array}{l}\text { South America } \\
\text { Tropical America }\end{array}$ & $\begin{array}{l}\text { Shrub } \\
\text { Tree }\end{array}$ \\
\hline 17. & Moraceae & Broussonetia papyrifera (Paper mulberry) & China & Tree \\
\hline
\end{tabular}


18. Myrtaceae

19. Onogranaceae

20. Poaceae

21. Pontederiaceae

22. Rubiaceae

23. Solanaceae

25. Verbenaceae
Eugenia uniflora (Surinam cherry) Psidium guajava (Apple guava)

Ludwigia peruviana (Peruvian primerose willow)

Arundo donax (Giant cane)

Imperata cylindrica (Cogon grass)

Paspalum vaginatum (Seashore paspalaum)

Pennisetum clandestinum (Kikuyu grass)

Phalaris arundinacea (Reed canary grass)

Spartina alterniflora (Smooth cord grass)

Eicchornia crassipes (Water hyacinth)

Coffea arabica (Arabic coffee)

C. canephora (Robust coffee)

Physalis peruviana (Cape gooseberry)

Solanum mauritianum (Wild tobacco tree)

Solanum sisymbriifolium (Tricky night shade)

Solanum viarum (Tropical soda apple)

Salvinia molesta (Water fern)

Lantana camara (Wild sage)
South America Tree

Cent. South America Tree

South America

Aquatic plant

Indian subcontinent Grass

Asia/Africa-doubtful Grass

North America Grass

Tropical Africa Grass

Europe Grass

South America Grass

South America Aquatic plant

Africa Shrub

Africa Shrub

South America Shrub

South America Tree

South America Herb

South America Shrub

South America Aquatic plant

Tropical America Shrub

Table 12: List of Designated Biosphere Reserves (Source: MoEF, 2012)

\begin{tabular}{|c|c|c|c|}
\hline S.No. & $\begin{array}{l}\text { Name and total geographical } \\
\text { area }\left(\mathrm{km}^{2}\right)\end{array}$ & $\begin{array}{l}\text { Date of } \\
\text { designation }\end{array}$ & Location in the States /UT \\
\hline 1. & Nilgiri (5520) & 01.08 .1986 & $\begin{array}{l}\text { Part of Wynad, Nagarhole, Bandipur and Madumalai, Nilambur, Silent Valley and } \\
\text { Siruvani hills (Tamil Nadu, Kerala and Karnataka) }\end{array}$ \\
\hline 2. & Nanda Devi (5860.69) & 18.01.1988 & Part of Chamoli, Pithoragarh and Almora Districts in Uttarakhand \\
\hline 3. & Nokrek (820) & 01.09 .1988 & Part of East, West and South Garo Hill districts in Meghalaya \\
\hline 4. & Manas (2837) & 14.03.1989 & $\begin{array}{l}\text { Part of Kokrajhar, Bongaigaon, Barpeta, Nalbari, Kamprup and Darang districts in } \\
\text { Assam }\end{array}$ \\
\hline 5. & Sunderban $(9630)$ & 29.03.1989 & Part of delta of Ganges and Brahamaputra river system in West Bengal \\
\hline 6. & Gulf of Mannar (10500) & 18.02.1989 & $\begin{array}{l}\text { Part of Gulf of Mannar extending from Rameswaram island in the North to } \\
\text { Kanyakumari in the South of Tamil Nadu }\end{array}$ \\
\hline 7. & Great Nicobar $(885)$ & 06.01 .1989 & Southern most island of Andaman and Nicobar Islands \\
\hline 8. & Similipal (4374) & 21.06.1994 & Part of Mayurbhanj district in Orissa \\
\hline 9. & Dibru-Saikhowa (765) & 28.07.1997 & Part of Dibrugarh and Tinsukia districts in Assam \\
\hline 10 . & Dehang Debang (5111.5) & 02.09 .1998 & Part of Upper Siang, West Siang and Dibang Valley districts in Arunachal Pradesh \\
\hline 11. & Pachmarhi (4981.72) & 03.03.1999 & Part of Betul, Hoshangabad and Chhindwara districts in Madhya Pradesh \\
\hline 12. & Khangchendzonga ( 2619.92 ) & 07.02 .2000 & Part of North and West districts in Sikkim \\
\hline 13. & Agasthyamalai (3500.36) & 12.11.2001 & $\begin{array}{l}\text { Part of Thirunelveli and Kanyakumari districts in Tamil Nadu and } \\
\text { Thiruvanthapuram, Kollam and Pathanmthitta districts in Kerala }\end{array}$ \\
\hline 14. & $\begin{array}{l}\text { Achanakmar-Amarkantak } \\
(3835.51)\end{array}$ & 30.03 .2005 & $\begin{array}{l}\text { Part of Anuppur and Dindori districts of Madhya Pradesh and Bilaspur district of } \\
\text { Chhattisgarh }\end{array}$ \\
\hline 15. & Kachchh $(12,454)$ & 29.01.2008 & Part of Kachchh, Rajkot, Surendranagar and Patan districts in Gujarat \\
\hline 16. & Cold Desert (7770) & 28.08.2009 & $\begin{array}{l}\text { Pin Valley National Park and surroundings; Chandratal and Sarchu; and Kibber } \\
\text { Wildlife sanctuary in Himachal Pradesh }\end{array}$ \\
\hline 17. & Seshachalam (4755.997) & 20.09.2010 & $\begin{array}{l}\text { Seshachalam hill ranges in Eastern Ghats encompassing part of Chittoor and } \\
\text { Kadapa districts in Andhra Pradesh }\end{array}$ \\
\hline 18. & Panna (2998.98) & 25.08 .2011 & Part of Pann and Chhattarpur districts in Madhya Pradesh \\
\hline
\end{tabular}


Table 13: Ramsar sites in India (26 Ramsar Sites, 689,131 hectares) (Source: http://www.ramsar.org/pdf/sitelist.pdf)

\begin{tabular}{|c|c|c|c|c|}
\hline Site & $\begin{array}{l}\text { Date of } \\
\text { designation }\end{array}$ & $\begin{array}{l}\text { Region, province, } \\
\text { state }\end{array}$ & Area & Coordinates \\
\hline Ashtamudi Wetland & $19 / 08 / 02$ & Kerala & 61,400 ha & $08^{\circ} 57^{\prime} \mathrm{N} 076^{\circ} 35^{\prime} \mathrm{E}$ \\
\hline Bhitarkanika Mangroves & $19 / 08 / 02$ & Orissa & 65,000 ha & $20^{\circ} 39^{\prime} \mathrm{N} 086^{\circ} 54^{\prime} \mathrm{E}$ \\
\hline Bhoj Wetland & $19 / 08 / 02$ & Madhya Pradesh & 3,201 ha & $23^{\circ} 14^{\prime} \mathrm{N} 077^{\circ} 20^{\prime} \mathrm{E}$ \\
\hline Chandertal Wetland & 08/11/05 & Himachal Pradesh & 49 ha & $32^{\circ} 29^{\prime} \mathrm{N} 077^{\circ} 36^{\prime} \mathrm{E}$ \\
\hline Chilika Lake & $01 / 10 / 81$ & Orissa & 116,500 ha & $19^{\circ} 42^{\prime} \mathrm{N} 085^{\circ} 21^{\prime} \mathrm{E}$ \\
\hline Deepor Beel & $19 / 08 / 02$ & Assam & 4,000 ha & $26^{\circ} 08^{\prime} \mathrm{N} 091^{\circ} 39^{\prime} \mathrm{E}$ \\
\hline East Calcutta Wetlands & $19 / 08 / 02$ & West Bengal & 12,500 ha & $22^{\circ} 27^{\prime} \mathrm{N} 088^{\circ} 27^{\prime} \mathrm{E}$ \\
\hline Harike Lake & $23 / 03 / 90$ & Punjab & 4,100 ha & $31^{\circ} 13^{\prime} \mathrm{N} 075^{\circ} 12^{\prime} \mathrm{E}$ \\
\hline Hokera Wetland & $08 / 11 / 05$ & Jammu and Kashmir & 1,375 ha & $34^{\circ} 05^{\prime} \mathrm{N} 074^{\circ} 42^{\prime} \mathrm{E}$ \\
\hline Kanjli & $22 / 01 / 02$ & Punjab & $183 \mathrm{ha}$ & $31^{\circ} 25^{\prime} \mathrm{N} 075^{\circ} 22^{\prime} \mathrm{E}$ \\
\hline Keoladeo National Park MR & $01 / 10 / 81$ & Rajasthan & 2,873 ha & $27^{\circ} 13^{\prime} \mathrm{N} 077^{\circ} 32^{\prime} \mathrm{E}$ \\
\hline Kolleru Lake & $19 / 08 / 02$ & Andhra Pradesh & 90,100 ha & $16^{\circ} 37^{\prime} \mathrm{N} 081^{\circ} 12^{\prime} \mathrm{E}$ \\
\hline Loktak Lake MR & $23 / 03 / 90$ & Manipur & 26,600 ha & $24^{\circ} 26^{\prime} \mathrm{N} 093^{\circ} 49^{\prime} \mathrm{E}$ \\
\hline Nalsarovar Bird Sanctuary & $24 / 09 / 12$ & Gujarat & 12,000 ha & $22^{\circ} 46^{\prime} 33^{\prime \prime} \mathrm{N} 072^{\circ} 02^{\prime} 21^{\prime \prime} \mathrm{E}$ \\
\hline Point Calimere Wildlife and Bird Sanctuary & $19 / 08 / 02$ & Tamil Nadu & 38,500 ha & $10^{\circ} 19^{\prime} \mathrm{N} 079^{\circ} 38^{\prime} \mathrm{E}$ \\
\hline Pong Dam Lake & $19 / 08 / 02$ & Himachal Pradesh & 15,662 ha & $32^{\circ} 01^{\prime} \mathrm{N} 076^{\circ} 05^{\prime} \mathrm{E}$ \\
\hline Renuka Wetland & $08 / 11 / 05$ & Himachal Pradesh & 20 ha & $31^{\circ} 37^{\prime} \mathrm{N} 077^{\circ} 27^{\prime} \mathrm{E}$ \\
\hline Ropar & $22 / 01 / 02$ & Punjab & 1,365 ha & $31^{\circ} 01^{\prime} \mathrm{N} 076^{\circ} 30^{\prime} \mathrm{E}$ \\
\hline Rudrasagar Lake & $08 / 11 / 05$ & Tripura & 240 ha & $23^{\circ} 29^{\prime} \mathrm{N} 090^{\circ} 01^{\prime} \mathrm{E}$ \\
\hline Sambhar Lake & $23 / 03 / 90$ & Rajasthan & 24,000 ha & $27^{\circ} 00^{\prime} \mathrm{N} 075^{\circ} 00^{\prime} \mathrm{E}$ \\
\hline Sasthamkotta Lake & $19 / 08 / 02$ & Kerala & 373 ha & $09^{\circ} 02^{\prime} \mathrm{N} 076^{\circ} 37^{\prime} \mathrm{E}$ \\
\hline Surinsar-Mansar Lakes & 08/11/05 & Jammu and Kashmir & 350 ha & $32^{\circ} 45^{\prime} \mathrm{N} 075^{\circ} 12^{\prime} \mathrm{E}$ \\
\hline Tsomoriri & $19 / 08 / 02$ & Jammu and Kashmir & 12,000 ha & $32^{\circ} 54^{\prime} \mathrm{N} 078^{\circ} 18^{\prime} \mathrm{E}$ \\
\hline Upper Ganga River (Brijghat to Narora Stretch) & 08/11/05 & Uttar Pradesh & 26,590 ha & $28^{\circ} 33^{\prime} \mathrm{N} 078^{\circ} 12^{\prime} \mathrm{E}$ \\
\hline Vembanad-Kol Wetland & $19 / 08 / 02$ & Kerala & 151,250 ha & $09^{\circ} 50^{\prime} \mathrm{N} 076^{\circ} 45^{\prime} \mathrm{E}$ \\
\hline Wular Lake & $23 / 03 / 90$ & Jammu and Kashmir & 18,900 ha & $34^{\circ} 16^{\prime} \mathrm{N} 074^{\circ} 33^{\prime} \mathrm{E}$ \\
\hline
\end{tabular}

MR: Sites included in the Montreux Record, "a record of Ramsar Sites where changes in ecological character have occurred, are occurring or are likely to occur" maintained by the Secretariat in consultation with the Contracting Party concerned (Recommendation 4.8). www.ramsar.org/montreux-record

flagship programmes for the conservation of tiger and elephant. Among the protected areas, India has designated 18 as Biosphere Reserves (BRs) in different parts of the country (Table 12) (MoEF, 2012). Out of these, so far seven, viz., Nilgiri, Gulf of Mannar, Sunderban, Nanda Devi, Pachmarhi, Similipal and Nokrek have been included in the World Network of Biosphere Reserves of UNESCO, also termed as 'world heritage sites'. In July 2016, Kangchendzonga National Park (KNP) in Sikkim has also been named as a world heritage site by the World Heritage Committee (WHC) of UNESCO. For India this is the first UNESCO 'mixed' heritage site exhibiting qualities of both natural and cultural significance. Recognizing the importance and significance of wetlands, India has established 26 Ramsar sites covering a surface area of 689,131 hectares (Table 13). Apart from establishing protected areas and Ramsar sites, a National Biodiversity Act was passed in 2002 and biodiversity rules were framed in 2004. A National Biodiversity Action Plan has also been released. 


\section{Acknowledgements}

J S S thanks INSA and R K C thanks CSIR for funding
support.

\section{References}

Banerjee L K and Ghosh D (1998) An Anthology of Indian Mangroves. pp 20-24, ENVIS Publication

Banerjee L K, Shastry A R K and Nayar M P (1989) Mangroves in India - Identification Manual. Botanical Survey of India

Bassi N, Kumar M D, Sharma A and Pardha-Saradhi P (2014) Status of wetlands in India: A review of extent, ecosystem benefits, threats and management strategies J Hydrol: Reg Stud 2 1-19

Biswas A (1984) Wetlands of India Water Qt Bull 13 15-35

Blankespoor B, Dasgupta S and Laplante B (2012) Sea-level Rise and Coastal Wetlands: Impacts and Costs. Policy Research Working Paper 6277. The World Bank

Champion H G and Seth S K (1968) A revised survey of the forest types of India. Government of India Publications

Chaturvedi R K and Raghubanshi AS (2013) Phenotypic plasticity in functional traits of woody species in tropical dry forest. In: Phenotypic Plasticity: Molecular Mechanisms, Evolutionary Significance and Impact on Speciation (Eds: Valentino J B and Harrelson P C) pp 35-66, Nova Science Publishers

Chaturvedi R K and Raghubanshi A S (2014) Species composition, distribution and diversity of woody species in tropical dry forest of India J Sustain Forest 33 729-756

Chaturvedi R K and Raghubanshi A S (2015) Assessment of carbon density and accumulation in mono- and multispecific stands in tropical dry forests of India For Ecol Manage 339 11-21

Chaturvedi R K and Raghubanshi A S (2016) Leaf life-span dynamics of woody species in tropical dry forests of India Trop Plant Res 3 199-212

Chaturvedi R K, Raghubanshi A S and Singh J S (2011a) Effect of small scale variations in environmental factors on the distribution of woody species in tropical deciduous forests of Vindhyan Highlands, India J Bot doi: 10.1155/2011/ 297097

Chaturvedi R K, Raghubanshi A S and Singh J S (2011b) Plant functional traits with particular reference to dry deciduous forests: A review J Biosc 36 963-981

Chaturvedi RK, Raghubanshi AS, and Singh JS (2011c) Carbon density and accumulation in woody species of tropical dry forest in India For Ecol Manage 262 1576-1588
Chaturvedi R K, Raghubanshi A S and Singh J S (2011d) Leaf attributes and tree growth in a tropical dry forest $J$ Veg Sci 22 917-931

Chaturvedi R K, Raghubanshi A S and Singh J S (2013) Growth of tree seedlings in a dry tropical forest in relation to soil moisture and leaf traits J Plant Ecol 6 158-170

Chaturvedi R K, Raghubanshi A S and Singh J S (2014) Relative effects of different leaf attributes on sapling growth in tropical dry forest $J$ Plant Ecol 7 544-558

Chaturvedi R K, Raghubanshi A S and Singh J S (2012) Effect of grazing and harvesting on diversity, recruitment and carbon accumulation of juvenile trees in tropical dry forests For Ecol Manage 284 152-162

Chaturvedi R K, Raghubanshi A S and Singh J S (2017a) Sapling harvest: A predominant factor affecting future compositionof tropical dry forests For Ecol Manage 384 221-235

Chaturvedi R K, Raghubashi A S, Tomlinson K W and Singh J S (2017b) Impacts of human disturbance in tropical dry forests increase with soil moisture stress $J$ Veg Sci doi: $10.1111 /$ jvs. 12547

Dabadghao P M and Shankarnarayan K A (1973) The Grass Cover of India. Indian Council of Agricultural Research

Das Gupta R and Shaw R (2013) Changing perspectives of mangrove management in India-An analytical overview Ocean Coast Manage 80 107-118

Deb S, Lynrah M M and Tiwari B K (2013) Technological innovations in shifting agricultural practices by three tribal farming communities of Meghalaya, northeast India Trop Ecol 54 133-148

FSI (2016) State of Forest Report. Forest Survey of India, Ministry of Environment

and Forests Government of India

Gopal B (2013) Water and biodiversity: an overview. In: The Wetlands Handbook.(Eds: Maltby E and Barker T) WileyBlackwell Publications

Gopal B (1987) Water Hyacinth. pp 471, Elsevier Science

Gopal B (1997) Biodiversity in inland aquatic ecosystems in India: An overview Int J Ecol Envir Sci 23 305-313

IUCN (2012) IUCN red list categories and criteria: Version 3.1. Second Edition. Gland, Switzerland and Cambridge, UK: IUCN

Jagtap T G (1994) Biodiversity in the Western Ghats: An Information Kit. pp 3-6. World Wide Fund, India and International Institute of Rural Reconstruction

Jha C S and Singh J S (1990) Composition and dynamics of dry 
tropical forest in relation to soil texture J Veg Sci 1 609614

Jha C S, Goparaju L, Tripathi A, Gharai B, Raghubanshi A S and Singh J S (2005) Forest fragmentation and its impact on species diversity: an analysis using remote sensing and GIS Biodivers Conserv 14 1681-1698

Jhingran A G (1991) Challenging frontiers in freshwater fisheries in India. In: Aqatic Sciences in India (Eds: Gopal B and Asthana V) pp 31-48, Indian Association for Limnology and Oceanography

Kenoyer LA (1921) Forest formations and successions in the Sat Tal Valley, Kumaun Himalayas J Indian Bot Soc $2236-258$

Kohli R K, Batish D R, Singh J S, Singh H P and Bhatt J R (2012) Plant invasion in India: an overview. In: Invasive alien plants: an ecological appraisal for the Indian subcontinent (Eds: Bhatt J R, Singh J S, Singh S P, Tripathi R S and Kohli R K) pp 1-9,CABI

MoEF (2009) India's fourth national report to the convention on biological diversity. Ministry of Environment and Forests, Government of India

MoEF (2012) Annual report 2011-12. Ministry of Environment and Forests, Government of India

Pandit M K and Grumbine R E (2012) Potential effects of ongoing and proposed hydropower development on terrestrial biological diversity in the Indian Himalaya Conserv Bio 26 1061-1071

Patel J G, Murthy T V R, Singh T S and Panigrahy S (2009) Analysis of the distribution pattern of wetlands in India in relation to climate change. In: Proceedings of the Workshop on Impact of Climate Change on Agriculture. Ahmedabad, India, 17-18 December (Eds: Panigrahy S, Shankar Ray S and Parihar J S) pp 282-287, International Society for Photogrammetry and Remote Sensing

Raghubanshi A S, Rai L C, Gaur J P and Singh J S (2005) Invasive alien species and biodiversity in India Curr Sci 88 539540

Ramakrishnan P S (1992) Shifting agriculture and sustainable development. Man and the Biosphere Series. Vol. 10. UNESCO-The Parthenon Publishing Group

Ramakrishnan P S (2015) Shifting agriculture and fallow management options. In: Shifting agriculture and environmental change: Indigenous people, agriculture and forest conservation (Ed: Cairns M) pp 186-198, Routledge

Ram R N, Rama Rao K V and Ghosh A (1994) Ramsar Sites of India: Chilika Lake. WWF India

Ranjan R and Upadhyay V P (1999) Ecological problems due to shifting cultivation Curr Sci 77 1246-1250
Rao K L (1975) India's Water Wealth. pp 255, Orient Longman

Rodgers W A, Panwar H S and Mathur V B (2000) Wildlife Protected Area Network in India: A review. Wildlife Institute of India

Roy P S, Joshi PK, Singh S, Agarwal S, Yadav D and Jegannathan C (2006) Biome mapping in India using vegetation type map derived using temporal satellite data and environmental parameters Ecolog Model 197 148-158

Sagar R and Singh J S (2004) Local plant species depletion in a tropical dry deciduous forest of northern India Environment Conserv 31 55-62

Sagar R and Singh J S (2005) Structure, diversity, and regeneration of tropical dry deciduous forest of northern India Biodivers Conserv 14 935-959

Saxena A K (1979) Ecology of vegetation complex of northwestern catchment of river Gola. Ph.D. Thesis, Kumaun University, Nainital

Selvam V (2003) Environmental classification of mangrove wetlands of India Curr Sci 84 757-765

Shillong Declaration (2004) Shifting Cultivation Regional Policy Dialogue Workshop for the Eastern Himalayas. Shillong, Meghalaya

Singh H T and Singh R K S (1994) Ramsar sites of India: Loktak lake Manipur. World Wide Fund for Nature

Singh J S and Singh K D (2011) Silviculture of dry deciduous forests, India. In: Silviculture in the Tropics (Eds: Günter S, Weber M, Stimm B and Mosandl R) pp273-283, Springer Verlag

Singh J S and Gupta S R (1993) Grasslands of southern Asia. In: Natural Grasslands: Eastern Hemisphere and Resume (Ed: Coupland R T) pp 83-123, Elsevier Science Publishers

Singh J S and Singh S P (1987) Forest vegetation of the Himalaya Bot Rev 53 81-192

Singh J S, Singh S P and Gupta S R (2015) Ecology, environmental science and conservation. S Chand and Company Pvt. Ltd.

Sugunan V V (1995) Reservoir Fishes of India. FAO Fisheries Technical Paper 345. pp 423, Food and Agriculture Organisation of the United Nations

Suryanarayanan N (1996) Environmental state-of-the-art of Indian lakes/reservoirs. In: Conservation and Management of Lakes/Reservoirs in India (Ed: Ando M) pp19-34, International Lake Environment Committee Foundation

Tiwari B K (2007) Shifting Agriculture-Spatiotemporal patterns and processes in North-Eastern India. In: Shifting Agriculture in Asia-Implications for Environmental 
Conservation and Sustainable Livelihood (Eds: Saxena K G, Liang L and Rerkasem K) pp 17-32, Bishen Singh Mahendra Pal Singh

Upadhyay V P, Ranjan R and Singh J S (2002) Human-mangrove conflicts: The way out Curr Sci $\mathbf{8 3}$ 1328-1336
Vijayan V S (1995) Keoladeo National Park. WWF-India, pp 72

Whyte R O (1968) Grasslands of the monsoon. Faber and Faber Yadava P S and Singh J S (1977) Grassland Vegetation: Its Structure, Function, Utilization and Management. pp 132, Today and Tomorrow's Printers and Publishers. 